\title{
BMC Cancer reviewer acknowledgement 2014
}

Dafne Solera

\section{Contributing reviewers}

The editors of BMC Cancer would like to thank all our reviewers who have contributed to the journal in Volume 14 (2014).

\begin{tabular}{|c|c|c|}
\hline $\begin{array}{l}\text { Philip Abbosh } \\
\text { USA }\end{array}$ & $\begin{array}{l}\text { Joachim Aerts } \\
\text { Netherlands }\end{array}$ & $\begin{array}{l}\text { Jennifer Aldrink } \\
\text { USA }\end{array}$ \\
\hline \multirow{2}{*}{$\begin{array}{l}\text { Daniel Abbott } \\
\text { USA }\end{array}$} & Ilir Agalliu & Agustí Alentorn \\
\hline & USA & Spain \\
\hline \multirow{2}{*}{$\begin{array}{l}\text { Sherif Abdel-Misih } \\
\text { USA }\end{array}$} & Kamran Aghayev & Riccardo Alessandro \\
\hline & USA & Italy \\
\hline \multirow{2}{*}{$\begin{array}{l}\text { Omar Abdel-Wahab } \\
\text { USA }\end{array}$} & Nishant Agrawal & Michael Alexandrakis \\
\hline & USA & Greece \\
\hline \multirow{2}{*}{$\begin{array}{l}\text { Takashige Abe } \\
\text { Japan }\end{array}$} & Yong Chan Ahn & Simak Ali \\
\hline & South Korea & UK \\
\hline \multirow{2}{*}{$\begin{array}{l}\text { Heinz-Harald Abholz } \\
\text { Germany }\end{array}$} & Hanjong Ahn & Sadeer Al-Kindi \\
\hline & South Korea & USA \\
\hline \multirow{2}{*}{$\begin{array}{l}\text { Roger Abounader } \\
\text { USA }\end{array}$} & Peter Ahn & Khawla Al-Kuraya \\
\hline & USA & Saudi Arabia \\
\hline \multirow{2}{*}{$\begin{array}{l}\text { Lucas Abrahao-Machado } \\
\text { Brazil }\end{array}$} & Myung-Ju Ahn & Hatim Allawi \\
\hline & South Korea & USA \\
\hline \multirow{2}{*}{$\begin{array}{l}\text { Filippo Acconcia } \\
\text { Italy }\end{array}$} & Koichi Aiura & Brian Allen \\
\hline & Japan & USA \\
\hline \multirow{2}{*}{$\begin{array}{l}\text { Paolo Accornero } \\
\text { Italy }\end{array}$} & Sally Akarolo-Anthony & Benjamin Allen \\
\hline & USA & USA \\
\hline \multirow{2}{*}{$\begin{array}{l}\text { Christopher Adams } \\
\text { USA }\end{array}$} & Rosemary Akhurst & Bryan Allen \\
\hline & USA & USA \\
\hline \multirow{2}{*}{$\begin{array}{l}\text { Raffaele Addeo } \\
\text { Italy }\end{array}$} & Bülent Arman Aksoy & Michael Allen \\
\hline & USA & UK \\
\hline \multirow{2}{*}{$\begin{array}{l}\text { Clement Adebamowo } \\
\text { USA }\end{array}$} & Andre Albergaria & Matthew Allen \\
\hline & Portugal & USA \\
\hline \multirow{2}{*}{$\begin{array}{l}\text { Prasad Adusumilli } \\
\text { USA }\end{array}$} & Scott Albert & Steven Allen \\
\hline & USA & UK \\
\hline \multicolumn{3}{|c|}{$\begin{array}{l}\text { Correspondence: Dafne.solera@biomedcentral.com } \\
\text { BioMed Central, Floor 6, } 236 \text { Gray's Inn Road, London WC1X 8HB, United } \\
\text { Kingdom }\end{array}$} \\
\hline \multicolumn{3}{|c|}{ 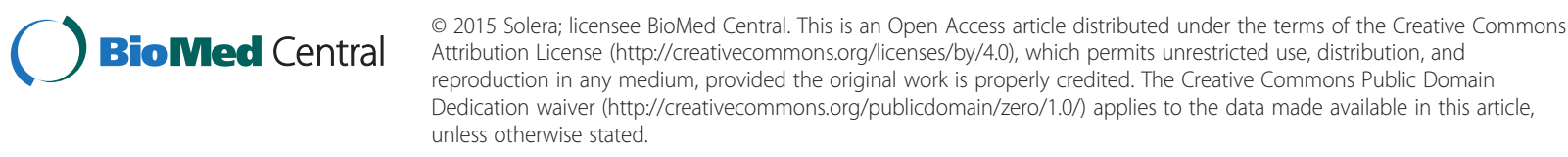 } \\
\hline
\end{tabular}


Gabriela Almeida
Portugal

Redhwan Al-Naggar

Malaysia

Vicente Alonso

Spain

Sergio Alonso

Spain

Robert Alston

UK

Sean Altekruse

USA

Begona Alvarez-Gonzalez USA

Antonio Alves

Brazil

Giulia Amadio

Italy

Larbi Amazit

France

Sreedhar Amere Subbarao India

Arm Amin

USA

Albert Amini

USA

Ziv Amir

UK

Ping An

USA

Hee An

South Korea

Ming-Wen An

USA

Ruopeng An

USA

Ioannis Anagnostopoulos

Germany

Satoshi Anai

Japan

Venkateshwari Ananthapur India

Dimitrios Anastasiou

UK
Berit Andersen

Denmark

Bogi Andersen

USA

Annie Anderson

UK

Benjamin Anderson

USA

Therese Andersson

Sweden

Bruno Andrade

Brazil

Victor Andrade

Brazil

Bengt Andrae

Sweden

Eran Andrechek

USA

Robert Andtbacka

USA

Mei-Kim Ang

Singapore

Jozef Anne

Belgium

Jean-Christophe Antoine

France

Alessandro Antonelli

Italy

Anji Anura

India

Daisuke Aoki

Japan

Lauren Aoude

Australia

Keishiro Aoyagi

Japan

Thomas Aparicio

France

Santiago Aparo

USA

Leonard Appleman

USA

Jack Arbiser

USA
Marc Arbyn

Belgium

Rosaria Arcone

Italy

Chantal Arditi

Switzerland

Diego Arenas

Mexico

Candy Arentz

USA

Paolo Aretini

Italy

David Argyle

UK

Saro Armenian

USA

Michael Arnold

USA

Manju Aron

USA

Amanda Arrington

USA

Hassan Arshad

USA

Robert Ashford

UK

Laura Ashley

UK

Jonathan Ashman USA

Luigi Aurisicchio

Italy

Philippe Autier

France

Romane Auvergne USA

Amir Avan

Netherlands

Kaamar Azijli

Netherlands

Hamdy Azim

Egypt

Amaya Azqueta

Spain 
Maria Azrad
USA

Ligia Azzalis

Brazil

Tsukasa Baba

Japan

Hideo Baba

Japan

Matthias Bache

Germany

Ulrike Bacher

Germany

Floor Backes

USA

Celia Badenas

Spain

Sunil Badve

USA

Young Kyung Bae

South Korea

Jeong-Heum Baek

South Korea

Swapnil Bagade

USA

Cedo Bagi

USA

Hilary Bagshaw

USA

Bruce Baguley

New Zealand

Simin Bahrami

USA

Li Bai

China

Longchuan Bai

USA

Tibor Bakacs

Hungary

Paul Bakaki

USA

Megan Baker

USA

Debora Balabram

Brazil
Tessa Balach

USA

Claudia Baldus

Germany

Marija Balic

Austria

Balint László Balint

Hungary

Karla Ballman

USA

Zsolt Balogi

USA

Fatima Baltazar

Portugal

Hamid Band

USA

Vimla Band

USA

Aria Baniahmad

Germany

Zhu Baosong

China

Massimo Barberis

Italy

Dario Barbone

USA

Nuno L Barbosa-Morais

Portugal

Stephane Bardet

France

Debmalya Barh

India

David Barker

USA

Michele Barone

Italy

Richard Barr

USA

Carlos Barrios

Brazil

Richard Barron

USA

João Diogo Barros-Silva

Portugal
Twyla Bartel

USA

Alakananda Basu

USA

Devraj Basu

USA

Sandip Basu

India

Madhumita Basu

USA

Ana Battastini

Brazil

Adi Baumgartner

UK

Alessandra Bearz

Italy

Francisco Beca

USA

Laurent Bedenne

France

Alicia Beeghly-Fadiel

USA

Juergen Behrens

Germany

Tim Beissbarth

Germany

Tanios Bekaii-Saab

USA

Antonino Belfiore

Italy

Cristiana Bellan

Italy

Joaquim Bellmunt USA

Qiwen Ben

China

Barbara Benassi

Italy

Offir Ben-Ishay

Israel

Lindsay Bennett

UK

Stephane Benoist

France 
David Bentrem
USA
Carmen Berasain
Spain

Guy Berchem

Luxembourg

Giovanni Luca Beretta

Italy

Bernhard Berger

Germany

Vance Berger

USA

Thierry Berghmans

Belgium

Lothar Bergmann

Germany

Johannes Berkhof

Netherlands

Dominique Bernard-Gallon

France

Rene Bernards

Netherlands

Sonja Berndt

USA

Gilbert Bernier

Canada

Alfredo Berruti

Italy

Donald Berry

USA

Tiffany Berry

USA

Francois Bertucci

France

Marianne Berwick

USA

Tomoko Betsuyaku

Japan

Kishor Bhakat

USA

Castigliano Bhamidipati

USA

Uddalak Bharadwaj

USA
Anant Bhatt

India

Bhaskar Bhattacharya

Singapore

Malay Bhattacharyya

India

Ujjal Bhawal

Japan

Nirmala Bhoo Pathy

Malaysia

Feng Bi

China

Long Bi

China

Giampaolo Bianchini

Italy

Irene Biasoli

Brazil

Kai Bickenbach

USA

Hemant Kumar Bid

USA

Francois-Clement Bidard

France

Siamak Bidel

Finland

Ettore Bidoli

Italy

Silvia Bielsa

Spain

Bernhard Biersack

Germany

Giuseppe Bifulco

Italy

Irene Bijnsdorp

Netherlands

Andreas Bikfalvi

France

Kate Lynn Bill

USA

Thomas Binder

Germany

Rebecca Birch

UK
Sarah Birken

USA

Eszter Biro

Hungary

Anders Bjartell

Sweden

Kimberly Blackwell

USA

Eric Blair

UK

Carmen Blanco-Aparicio

Spain

Justin Blasberg

USA

Boris Blechacz

USA

Matthew Block

USA

Elisabeth Bloemena

Netherlands

Karen Blyth

UK

Zhai Bo

China

Gerd Bobe

USA

Carla Boccaccio

Italy

Udo Bode

Germany

Clara Bodelon

USA

Martin Bohac

Slovakia

Antonio Boing

Brazil

Richard Bold

USA

Jesper Bonde

Denmark

Edith Bonnelye

France

Stefanos Bonovas

Greece 
Stephanie Boone

USA

Jan Bornschein

Germany

David Borowski

UK

Pedro Borralho

Portugal

Josep Borras

Spain

Stefania Bortoluzzi Italy

Silvano Bosari

Italy

Fred Bosman

Switzerland

Paolo Bossi

Italy

Jan Bouchal

Czech Republic

Sotiria Boukouvala

Greece

Liam Bourke

UK

Anne-Marie Bouvier

France

Nathan Bowen

USA

John Boyages

Australia

Sabrina Boyrie

USA

Serdar Bozdag

USA

Alexandre Bozec

France

Katja Irene Braam

Netherlands

Edward Bradley

Canada

Lucia Braga

Brazil

Elena Ioana Braicu

Germany
Simone Braig

Germany

Kate Brain

UK

Mariana Brait

USA

Mirjana Brankovic-Magic

Serbia

Maria Braoudaki

Greece

Theodore Brasky

USA

Isabelle Bray

UK

Patrick Brest

France

Guy Brock

USA

Inka Brockhausen

Canada

Giuseppe Bronte

Italy

Reuben Broom

New Zealand

James Broome

USA

Christine Brostjan

Austria

Mick Brown

UK

Terry Brown

UK

Anthony Bruce

Canada

Adam Brufsky

USA

Matteo Brunelli

Italy

Lauro Bucchi

Italy

Jan Budczies

Germany

Laurien Maria Buffart

Netherlands
Olivier Buhard

France

Ralph Bundschuh

Germany

Emily Burger

Norway

Barbara Burtness

USA

Rita Busuttil

Australia

Sebastiano Buti

Italy

Lisa Butler

Australia

Alison Butt

Australia

Maria Cabanillas

USA

Hale Basak Caglar

Turkey

Mu-Yan Cai

China

Sanjun Cai

China

Dawei Cai

USA

Hong Cai

China

Silvia Calabuig-Fariñas

Spain

Natalia Calanzani

UK

George Calin

USA

Gloria Callard

USA

Federica Calore

USA

Enrica Calura

Italy

Felipe Calvo

Spain

E. Ramsay Camp

USA 


\begin{tabular}{|c|c|c|}
\hline Kerry Campbell & Luis Carvajal Carmona & Suzanne Chambers \\
\hline USA & USA & Australia \\
\hline Michael Campbell & Beatriz Carvalho & Michael Chan \\
\hline USA & Netherlands & Taiwan \\
\hline Kevin Camphausen & Joana Carvalho & Jennifer Chan \\
\hline USA & Portugal & USA \\
\hline Giuseppina Campisi & Ivan Casaburi & Jennifer Chan \\
\hline Italy & Italy & Canada \\
\hline Nicole Campos & Silvia Casadei & Karen Chan \\
\hline USA & USA & Hong Kong \\
\hline Francisco Candido Dos Reis & Emilio Casanova & Michael Chan \\
\hline Brazil & Austria & USA \\
\hline Qing Cao & Patricia Casbas-Hernandez & Ashwini Chand \\
\hline China & USA & Australia \\
\hline Guangwen Cao & Bruce Case & Alice Y.W. Chang \\
\hline China & Canada & Taiwan \\
\hline Wenqing Cao & Alejandra Castanon & Chien-Chung Chang \\
\hline USA & UK & Taiwan \\
\hline Gabriel Capella & Daniel Castellano & Jong Hee Chang \\
\hline Spain & Spain & South Korea \\
\hline Marzia Capelletti & Isabella Castellano & Kuo-Wei Chang \\
\hline USA & Italy & Tanzania \\
\hline Vera Cappelletti & Gabriella Castoria & Yu-Chao Chang \\
\hline Italy & Italy & Taiwan \\
\hline Emilia Caputo & Stefania Catalano & Kai-Ping Chang \\
\hline Italy & Italy & Taiwan \\
\hline Michele Caraglia & Chelsea Catsburg & Hong Chang \\
\hline Italy & USA & Canada \\
\hline Eugen Carasevici & Erika Cecchin & Yu-Sun Chang \\
\hline Romania & Italy & Taiwan \\
\hline Romilda Cardin & Francesco Cellini & Yoon Soo Chang \\
\hline Italy & Italy & South Korea \\
\hline Vinicio Carloni & Lorenzo Ceppi & Wu Changjun \\
\hline Italy & USA & China \\
\hline Francisco Javier Carmona Sanz & Giovanni Luca Ceresoli & Jui-I Chao \\
\hline USA & Italy & Taiwan \\
\hline Shamus Carr & Hyuk-Jin Cha & Kathy Chapman \\
\hline USA & South Korea & Australia \\
\hline Dirce Carraro & Karl Chai & Bjoern Chapuy \\
\hline Brazil & USA & USA \\
\hline Jason Carroll & Arthit Chairoungdua & Al Charest \\
\hline UK & Thailand & USA \\
\hline Rosangela Caruso & Marc Chamberlain & Ayana Chase \\
\hline Italy & USA & USA \\
\hline
\end{tabular}




\begin{tabular}{|c|c|c|}
\hline $\begin{array}{l}\text { Arun Chauhan } \\
\text { USA }\end{array}$ & $\begin{array}{l}\text { Yongwen Chen } \\
\text { China }\end{array}$ & $\begin{array}{l}\text { Yolanda I Chirino } \\
\text { Mexico }\end{array}$ \\
\hline $\begin{array}{l}\text { Alcides Chaux } \\
\text { Paraguay }\end{array}$ & $\begin{array}{l}\text { Zong-You Chen } \\
\text { China }\end{array}$ & $\begin{array}{l}\text { Tai-Jan Chiu } \\
\text { Taiwan }\end{array}$ \\
\hline $\begin{array}{l}\text { Srikumar Chellappan } \\
\text { USA }\end{array}$ & $\begin{array}{l}\text { Chao-Wen Cheng } \\
\text { Taiwan }\end{array}$ & $\begin{array}{l}\text { Sherry Yueh-Hsia Chiu } \\
\text { Taiwan }\end{array}$ \\
\hline $\begin{array}{l}\text { Mu-Kuan Chen } \\
\text { Taiwan }\end{array}$ & $\begin{array}{l}\text { Ting-Yuan Cheng } \\
\text { USA }\end{array}$ & $\begin{array}{l}\text { Miguel Chiurillo } \\
\text { Venezuela }\end{array}$ \\
\hline $\begin{array}{l}\text { Ching-Shih Chen } \\
\text { USA }\end{array}$ & $\begin{array}{l}\text { Jason Chia-Hsien Cheng } \\
\text { Taiwan }\end{array}$ & $\begin{array}{l}\text { Sang-Hee Cho } \\
\text { South Korea }\end{array}$ \\
\hline $\begin{array}{l}\text { Chunhua Chen } \\
\text { China }\end{array}$ & $\begin{array}{l}\text { Steven Cheng } \\
\text { China }\end{array}$ & $\begin{array}{l}\text { Yung Hyun Choi } \\
\text { South Korea }\end{array}$ \\
\hline $\begin{array}{l}\text { Chuang Chen } \\
\text { China }\end{array}$ & $\begin{array}{l}\text { Dong-Joo Cheon } \\
\text { USA }\end{array}$ & $\begin{array}{l}\text { Kyung-Chul Choi } \\
\text { South Korea }\end{array}$ \\
\hline $\begin{array}{l}\text { Chang-Han Chen } \\
\text { Taiwan }\end{array}$ & $\begin{array}{l}\text { Jaehun Cheong } \\
\text { South Korea }\end{array}$ & $\begin{array}{l}\text { Kui Son Choi } \\
\text { South Korea }\end{array}$ \\
\hline $\begin{array}{l}\text { Ming Chen } \\
\text { China }\end{array}$ & $\begin{array}{l}\text { Sok Ching Cheong } \\
\text { Malaysia }\end{array}$ & $\begin{array}{l}\text { Lydia Choi } \\
\text { USA }\end{array}$ \\
\hline $\begin{array}{l}\text { Wan Qing Chen } \\
\text { China }\end{array}$ & $\begin{array}{l}\text { Radostina Cherneva } \\
\text { Bulgaria }\end{array}$ & $\begin{array}{l}\text { Won-Il Choi } \\
\text { South Korea }\end{array}$ \\
\hline $\begin{array}{l}\text { Zhe-Sheng Chen } \\
\text { USA }\end{array}$ & $\begin{array}{l}\text { Chandramu Chetty } \\
\text { USA }\end{array}$ & $\begin{array}{l}\text { Ravi Chokshi } \\
\text { USA }\end{array}$ \\
\hline $\begin{array}{l}\text { Tse-Ching Chen } \\
\text { Taiwan }\end{array}$ & $\begin{array}{l}\text { Arthur Cheung } \\
\text { Hong Kong }\end{array}$ & $\begin{array}{l}\text { Geoffrey Chong } \\
\text { Australia }\end{array}$ \\
\hline $\begin{array}{l}\text { Honglei Chen } \\
\text { China }\end{array}$ & $\begin{array}{l}\text { Felix Cheung } \\
\text { USA }\end{array}$ & $\begin{array}{l}\text { Su Pin Choo } \\
\text { Singapore }\end{array}$ \\
\hline $\begin{array}{l}\text { James Chen } \\
\text { USA }\end{array}$ & $\begin{array}{l}\text { Karen Cheung } \\
\text { Canada }\end{array}$ & $\begin{array}{l}\text { Helene Choquet } \\
\text { USA }\end{array}$ \\
\hline $\begin{array}{l}\text { Jeon-Hor Chen } \\
\text { USA }\end{array}$ & $\begin{array}{l}\text { Siu Tim Cheung } \\
\text { Hong Kong }\end{array}$ & $\begin{array}{l}\text { Teh-Ying Chou } \\
\text { Taiwan }\end{array}$ \\
\hline $\begin{array}{l}\text { Jianyong Chen } \\
\text { USA }\end{array}$ & $\begin{array}{l}\text { Chun-Ju Chiang } \\
\text { Taiwan }\end{array}$ & $\begin{array}{l}\text { Kuan-Chih Chow } \\
\text { Taiwan }\end{array}$ \\
\hline $\begin{array}{l}\text { Kuen-Feng Chen } \\
\text { Taiwan }\end{array}$ & $\begin{array}{l}\text { Hung-Che Chiang } \\
\text { Taiwan }\end{array}$ & $\begin{array}{l}\text { Louis Chow } \\
\text { Hong Kong }\end{array}$ \\
\hline $\begin{array}{l}\text { Ying Chen } \\
\text { China }\end{array}$ & $\begin{array}{l}\text { Laura Chiecchio } \\
\text { UK }\end{array}$ & $\begin{array}{l}\text { Balram Chowbay } \\
\text { Singapore }\end{array}$ \\
\hline $\begin{array}{l}\text { Wu-Fu Chen } \\
\text { Taiwan }\end{array}$ & $\begin{array}{l}\text { Chih-Yen Chien } \\
\text { Taiwan }\end{array}$ & $\begin{array}{l}\text { Edwin Choy } \\
\text { USA }\end{array}$ \\
\hline $\begin{array}{l}\text { Zhiyi Chen } \\
\text { China }\end{array}$ & $\begin{array}{l}\text { Lung-Chang Chien } \\
\text { USA }\end{array}$ & $\begin{array}{l}\text { Jesper Frank Christensen } \\
\text { Denmark }\end{array}$ \\
\hline $\begin{array}{l}\text { Xinbin Chen } \\
\text { USA }\end{array}$ & $\begin{array}{l}\text { Yoshitomo Chihara } \\
\text { Japan }\end{array}$ & $\begin{array}{l}\text { Saranya Chumsri } \\
\text { USA }\end{array}$ \\
\hline $\begin{array}{l}\text { Yongshun Chen } \\
\text { China }\end{array}$ & $\begin{array}{l}\text { Giovanna Chiorino } \\
\text { Italy }\end{array}$ & $\begin{array}{l}\text { Kuo-Piao Chung } \\
\text { Taiwan }\end{array}$ \\
\hline
\end{tabular}


Yeun-Jun Chung

South Korea

Younh-Hwa Chung

South Korea

Fabio Cianchi

Italy

Amelia Cimmino

Italy

Daniel Ciocca

Argentina

Paola Ciriaco

Italy

Frank Claessens

Belgium

Andrew Clamp

UK

Jennifer Clarke

USA

Charles Clevenger

USA

Hiram Cody

USA

Kelly Coffey

UK

Mark Cohen

USA

Bertrand Coiffier

France

Gisele Colleoni

Brazil

Marco Colleoni

Italy

Luis Colomo

Spain

Emile Comans

Netherlands

Niamh Conlon

USA

Nathan Consedine

New Zealand

Alfredo Conti

Italy

William Omar Contreras Lopez

Colombia
Giovanni Conzo

Italy

Darrel Cook

Canada

Kumarasen Cooper

USA

Wendy Cooper

Australia

Rob Coppes

Netherlands

Pierre Cordelier

France

Seth Joel Corey

USA

Giacomo Corrado

Italy

Mark Corrigan

Ireland

Javier Cortes Bordoy

Spain

Giuliana Cortese

Italy

Laura Cortesi

Italy

Renzo Corvo

Italy

Silvia Liliana Cossio

Brazil

Frederico Costa

Brazil

Jose Luis Costa

Portugal

Vera Costa

Portugal

Massimo Costantini

Italy

Gregory Cote

USA

Paul Cottu

France

Fergus Couch

USA

David Countryman

USA
Johannes Coy

Germany

Susanna Cramb

Australia

Ross Cranston

USA

S. Michael Crawford

UK

Sara Cresta

Italy

Carmen Criscitiello

Italy

Cinzia Crivellaro

Italy

Alysha Croker

Canada

Colleen Croniger

USA

Tim Crook

UK

Nina Crowley

USA

Ofelia Cruz

Spain

Vincent Cryns

USA

Joaquin Cubiella

Spain

Alessandro Cucchetti

Italy

Ioan Cucoranu

USA

Ziyou Cui

USA

S. Nicole Culos-Reed

Canada

Jeff Cummings

UK

Antonio Cuneo

Italy

Margaret Currie

New Zealand

Karen Curtin

USA 


\begin{tabular}{|c|c|c|}
\hline Kate Cuschieri & Sanford Dawsey & Metin Demirkaya \\
\hline UK & USA & Turkey \\
\hline Vahid Reza Dabbagh Kakhki & Heather Dawson & Semra Demokan \\
\hline Iran & Switzerland & Turkey \\
\hline Gabi U Dachs & Jonathan Day & An-Mei Deng \\
\hline New Zealand & USA & China \\
\hline Alv A. Dahl & Prithwish De & Dajun Deng \\
\hline Norway & Canada & China \\
\hline Liping Dai & Francesca De Amicis & Zhao-Qun Deng \\
\hline USA & Italy & China \\
\hline Adrien Daigeler & Michele De Bortoli & Carsten Denkert \\
\hline Germany & Italy & Germany \\
\hline Maria Dalamaga & Filippo De Braud & Lynette Denny \\
\hline Greece & Italy & South Africa \\
\hline Katiuscia Dallaglio & Patricia De Cremoux & Susan Dent \\
\hline Italy & France & Canada \\
\hline Christopher Daly & Pierre De Delva & Elena Deryugina \\
\hline USA & USA & USA \\
\hline Nadia Dandachi & Sonia De Francesco & Anjali Deshpande \\
\hline Austria & Italy & USA \\
\hline Mark Danese & Valli De Re & Beena Cr Devi \\
\hline USA & Italy & Malaysia \\
\hline Cyril Danjoux & Silvia De Sanjose & Sarah Dewilde \\
\hline Canada & Spain & Belgium \\
\hline Silvia Darb-Esfahani & Ines De Santiago & Nandini Dey \\
\hline Germany & UK & USA \\
\hline Özlem Darcansoy Iseri & Paulo De Sepulveda & Subhojit Dey \\
\hline Turkey & France & India \\
\hline Kakoli Das & Marco De Velasco & Saravana Dhanasekaran \\
\hline Singapore & Japan & USA \\
\hline Mukul Das & Henry John Christiaan De Vries & Debanjan Dhar \\
\hline India & Netherlands & USA \\
\hline Kaustubh Datta & Olivier De Wever & Santosh Dhule \\
\hline USA & Belgium & USA \\
\hline Heena Dave & Alberto Deganello & Pietro Di Fazio \\
\hline USA & Italy & Germany \\
\hline Ben Davidson & Tim Dekker & Giuseppe Di Lorenzo \\
\hline Norway & Netherlands & Italy \\
\hline Guido Davidzon & Mario Del Rosso & Massimo Di Maio \\
\hline USA & Italy & Italy \\
\hline Elizabeth Davies & Michael Dellinger & Luca Di Tommaso \\
\hline UK & USA & Italy \\
\hline Jean-Luc Davignon & Wendy Demark-Wahnefried & Eleftherios Diamandis \\
\hline France & USA & Canada \\
\hline
\end{tabular}




\begin{tabular}{|c|c|c|}
\hline $\begin{array}{l}\text { Sérgio Dias } \\
\text { Portugal }\end{array}$ & $\begin{array}{l}\text { Michael Dixon } \\
\text { UK }\end{array}$ & $\begin{array}{l}\text { Richard Drake } \\
\text { USA }\end{array}$ \\
\hline $\begin{array}{l}\text { Stefan Diem } \\
\text { Switzerland }\end{array}$ & $\begin{array}{l}\text { Hooman Djaladat } \\
\text { USA }\end{array}$ & $\begin{array}{l}\text { Didier Dreau } \\
\text { USA }\end{array}$ \\
\hline $\begin{array}{l}\text { Veronique Dieras } \\
\text { France }\end{array}$ & $\begin{array}{l}\text { Mehdi Djekidel } \\
\text { USA }\end{array}$ & $\begin{array}{l}\text { Paul Drew } \\
\text { Australia }\end{array}$ \\
\hline $\begin{array}{l}\text { Jill Dietz } \\
\text { USA }\end{array}$ & $\begin{array}{l}\text { Khanh Do } \\
\text { USA }\end{array}$ & $\begin{array}{l}\text { Gypsyamber D'Souza } \\
\text { USA }\end{array}$ \\
\hline $\begin{array}{l}\text { Analisa Difeo } \\
\text { USA }\end{array}$ & $\begin{array}{l}\text { Alexander Dobrovic } \\
\text { Australia }\end{array}$ & $\begin{array}{l}\text { Jiajun Du } \\
\text { China }\end{array}$ \\
\hline $\begin{array}{l}\text { Ekkehard Dikomey } \\
\text { Germany }\end{array}$ & $\begin{array}{l}\text { Epameinondas Dogeas } \\
\text { USA }\end{array}$ & $\begin{array}{l}\text { Haifeng Duan } \\
\text { China }\end{array}$ \\
\hline $\begin{array}{l}\text { Mary Dillhoff } \\
\text { USA }\end{array}$ & $\begin{array}{l}\text { Jennifer Doherty } \\
\text { USA }\end{array}$ & $\begin{array}{l}\text { Shiwei Duan } \\
\text { China }\end{array}$ \\
\hline $\begin{array}{l}\text { Gabriel Dimattia } \\
\text { Canada }\end{array}$ & $\begin{array}{l}\text { Ramona Dolscheid-Pommerich } \\
\text { Germany }\end{array}$ & $\begin{array}{l}\text { Tao (Tony) Duan } \\
\text { China }\end{array}$ \\
\hline $\begin{array}{l}\text { Johannes Carl Athanasios Dimopoulos } \\
\text { Greece }\end{array}$ & $\begin{array}{l}\text { Gemma Dominguez } \\
\text { Spain }\end{array}$ & $\begin{array}{l}\text { Shikha Dubey } \\
\text { India }\end{array}$ \\
\hline $\begin{array}{l}\text { Goberdhan Dimri } \\
\text { USA }\end{array}$ & $\begin{array}{l}\text { Davide Maria Donati } \\
\text { Italy }\end{array}$ & $\begin{array}{l}\text { Anna Dubrovska } \\
\text { Germany }\end{array}$ \\
\hline $\begin{array}{l}\text { Pei-Rong Ding } \\
\text { China }\end{array}$ & $\begin{array}{l}\text { Nicholas Donato } \\
\text { USA }\end{array}$ & $\begin{array}{l}\text { Francois Ducray } \\
\text { France }\end{array}$ \\
\hline $\begin{array}{l}\text { Wen-Xing Ding } \\
\text { USA }\end{array}$ & $\begin{array}{l}\text { Jia-Hong Dong } \\
\text { China }\end{array}$ & $\begin{array}{l}\text { Vikas Dudeja } \\
\text { USA }\end{array}$ \\
\hline $\begin{array}{l}\text { Lijun Ding } \\
\text { China }\end{array}$ & $\begin{array}{l}\text { Qihan Dong } \\
\text { Australia }\end{array}$ & $\begin{array}{l}\text { Maire Duggan } \\
\text { Canada }\end{array}$ \\
\hline $\begin{array}{l}\text { Marina Diniz } \\
\text { Brazil }\end{array}$ & $\begin{array}{l}\text { Xiaojing Dong } \\
\text { China }\end{array}$ & $\begin{array}{l}\text { Pierre-Antoine Dugué } \\
\text { Denmark }\end{array}$ \\
\hline $\begin{array}{l}\text { Andreas Dinkel } \\
\text { Germany }\end{array}$ & $\begin{array}{l}\text { Yuan Dongmei } \\
\text { China }\end{array}$ & $\begin{array}{l}\text { Lucien Duijm } \\
\text { Netherlands }\end{array}$ \\
\hline $\begin{array}{l}\text { Peter Dipasco } \\
\text { USA }\end{array}$ & $\begin{array}{l}\text { Joseph Dort } \\
\text { Canada }\end{array}$ & $\begin{array}{l}\text { Martine Dunnwald } \\
\text { USA }\end{array}$ \\
\hline $\begin{array}{l}\text { Bahar Dirican } \\
\text { Turkey }\end{array}$ & $\begin{array}{l}\text { C George Priya Doss } \\
\text { India }\end{array}$ & $\begin{array}{l}\text { Francine Durocher } \\
\text { Canada }\end{array}$ \\
\hline $\begin{array}{l}\text { Kristin Dittmar } \\
\text { USA }\end{array}$ & $\begin{array}{l}\text { Isabel Dos Santos Silva } \\
\text { UK }\end{array}$ & $\begin{array}{l}\text { Adriana Dusso } \\
\text { Spain }\end{array}$ \\
\hline $\begin{array}{l}\text { Thomas Dittmar } \\
\text { Germany }\end{array}$ & $\begin{array}{l}\text { Maura Dowling } \\
\text { Ireland }\end{array}$ & $\begin{array}{l}\text { Janice Dutcher } \\
\text { USA }\end{array}$ \\
\hline $\begin{array}{l}\text { Francesco Dituri } \\
\text { Italy }\end{array}$ & $\begin{array}{l}\text { Amy Downing } \\
\text { UK }\end{array}$ & $\begin{array}{l}\text { Amit Dutt } \\
\text { India }\end{array}$ \\
\hline $\begin{array}{l}\text { Leo Ditzel Filho } \\
\text { USA }\end{array}$ & $\begin{array}{l}\text { Jerome Doyen } \\
\text { France }\end{array}$ & $\begin{array}{l}\text { Roisin Dwyer } \\
\text { Ireland }\end{array}$ \\
\hline $\begin{array}{l}\text { Tayyab Diwan } \\
\text { USA }\end{array}$ & $\begin{array}{l}\text { Tommaso Dragani } \\
\text { Italy }\end{array}$ & $\begin{array}{l}\text { Lars Dyrskjøt } \\
\text { Denmark }\end{array}$ \\
\hline
\end{tabular}


Piotr Dziegiel

Poland

Peter Dziegielewski

USA

Alan Eastman
USA
Jeanette Eckel-Passow
USA

Iris Eder

Austria

Anders Edsjö

Sweden

Dylan Edwards

UK

Joanne Edwards

UK

Mary Egan

Canada

Marcelo Ehrlich

Israel

Pieter Eichhorn

Singapore

Günter Eisele

Switzerland

Wolfgang Eisterer

Austria

Ram Eitan

Israel

Lars Ekblad

Sweden

Fathia El Sharkawi

Egypt

Samer El-Dika

USA

Olivier Elemento

USA

Daniel Elias

Ethiopia

Rossella Elisei

Italy

Lesley Ellies

USA

Jacqueline Ellis

UK
Jean Anderson Eloy

USA

J. Mark Elwood

New Zealand

Cynthia Emory

USA

Agamemnon Epenetos

UK

Lisa Ercolano

USA

Costantino Errani

Italy

Cam Escoffery

USA

Hannu Eskola

Finland

Jose M. Estrela

Spain

Arnold Etame

USA

Joseph Evans

USA

Matthias Evert

Germany

Eduardo Eyras

Spain

Antonio Facchiano

Italy

Johannes Fahrmann

USA

Stefano Fais

Italy

Jean Faivre

France

Marwan Fakih

USA

Zhining Fan

China

Jia Fan

China

Lifang Fan

China

Meiyun Fan

USA
Xing Fan

USA

Bingliang Fang

USA

Fu-Min Fang

Taiwan

Qingming Fang

USA

Xiao Fang

China

Jia Fang

USA

Xiaoguang Fang

USA

Eduardo Farias

USA

Lucyana Farias

Brazil

Mohamad Farid

Singapore

Ahmad Faried

Japan

Fabio Farinati

Italy

Daniel Farkas

USA

Rosa Farràs

Spain

Peter Fasching

Germany

Ambrogio Fassina

Italy

Elvira Favoino

Italy

Jerome Fayette

France

Veronika Fedirko

USA

Manuel Felices

Sweden

Jorge Felix

Portugal

Richard Feltbower

UK 


\begin{tabular}{|c|c|c|}
\hline $\begin{array}{l}\text { Angelo Pietro Femia } \\
\text { Italy }\end{array}$ & $\begin{array}{l}\text { Uta Flucke } \\
\text { Netherlands }\end{array}$ & $\begin{array}{l}\text { Rafael Fridman } \\
\text { USA }\end{array}$ \\
\hline $\begin{array}{l}\text { Nicola Fenderico } \\
\text { Italy }\end{array}$ & $\begin{array}{l}\text { Emmanouil Fokas } \\
\text { Germany }\end{array}$ & $\begin{array}{l}\text { Philip Friedlander } \\
\text { USA }\end{array}$ \\
\hline $\begin{array}{l}\text { Annika Fendler } \\
\text { Germany }\end{array}$ & $\begin{array}{l}\text { Maria Aparecida A Koike Folgueira } \\
\text { Brazil }\end{array}$ & $\begin{array}{l}\text { Daniel Frigo } \\
\text { USA }\end{array}$ \\
\hline $\begin{array}{l}\text { Hanping Feng } \\
\text { USA }\end{array}$ & $\begin{array}{l}\text { Marco Folini } \\
\text { Italy }\end{array}$ & $\begin{array}{l}\text { Jan Frisell } \\
\text { Sweden }\end{array}$ \\
\hline $\begin{array}{l}\text { Lynnette Robin Ferguson } \\
\text { New Zealand }\end{array}$ & $\begin{array}{l}\text { Gunnar Folprecht } \\
\text { Germany }\end{array}$ & $\begin{array}{l}\text { Holger Fröhlich } \\
\text { Germany }\end{array}$ \\
\hline $\begin{array}{l}\text { Almudena Fernandez Briera } \\
\text { Spain }\end{array}$ & $\begin{array}{l}\text { Kam Weng Fong } \\
\text { Singapore }\end{array}$ & $\begin{array}{l}\text { Olivia Fromigue } \\
\text { France }\end{array}$ \\
\hline $\begin{array}{l}\text { Arias Fernando } \\
\text { Spain }\end{array}$ & $\begin{array}{l}\text { Laia Font } \\
\text { Spain }\end{array}$ & $\begin{array}{l}\text { Michael Frumovitz } \\
\text { USA }\end{array}$ \\
\hline $\begin{array}{l}\text { Mårten Fernö } \\
\text { Sweden }\end{array}$ & $\begin{array}{l}\text { Caterina Fontanella } \\
\text { Germany }\end{array}$ & $\begin{array}{l}\text { Shen Fu } \\
\text { China }\end{array}$ \\
\hline $\begin{array}{l}\text { Cristina Ferrás } \\
\text { Portugal }\end{array}$ & $\begin{array}{l}\text { Lindsay Forbes } \\
\text { UK }\end{array}$ & $\begin{array}{l}\text { Loning Fu } \\
\text { USA }\end{array}$ \\
\hline $\begin{array}{l}\text { Irene Ferrer } \\
\text { Spain }\end{array}$ & $\begin{array}{l}\text { Marvella Ford } \\
\text { USA }\end{array}$ & $\begin{array}{l}\text { Siqing Fu } \\
\text { USA }\end{array}$ \\
\hline $\begin{array}{l}\text { Yonatan Feuermann } \\
\text { Germany }\end{array}$ & $\begin{array}{l}\text { Charles Forscher } \\
\text { USA }\end{array}$ & $\begin{array}{l}\text { Xianghui Fu } \\
\text { USA }\end{array}$ \\
\hline $\begin{array}{l}\text { Mary Jo Fidler } \\
\text { USA }\end{array}$ & $\begin{array}{l}\text { Paul Foster } \\
\text { UK }\end{array}$ & $\begin{array}{l}\text { Xiao-Long Fu } \\
\text { China }\end{array}$ \\
\hline $\begin{array}{l}\text { Ryan Fields } \\
\text { USA }\end{array}$ & $\begin{array}{l}\text { Julie Fradette } \\
\text { Canada }\end{array}$ & $\begin{array}{l}\text { Fumihiko Fujita } \\
\text { Japan }\end{array}$ \\
\hline $\begin{array}{l}\text { Joana Figueiredo } \\
\text { Portugal }\end{array}$ & $\begin{array}{l}\text { Silvia Franceschi } \\
\text { France }\end{array}$ & $\begin{array}{l}\text { Kazuya Fukuoka } \\
\text { Japan }\end{array}$ \\
\hline $\begin{array}{l}\text { Jorge Filmus } \\
\text { Canada }\end{array}$ & $\begin{array}{l}\text { Valerie Francescutti } \\
\text { Canada }\end{array}$ & $\begin{array}{l}\text { Ada Funaro } \\
\text { Italy }\end{array}$ \\
\hline $\begin{array}{l}\text { Jeffrey Fine } \\
\text { USA }\end{array}$ & $\begin{array}{l}\text { Renato Franco } \\
\text { Italy }\end{array}$ & $\begin{array}{l}\text { Pauline Funchain } \\
\text { USA }\end{array}$ \\
\hline $\begin{array}{l}\text { Daniel Fink } \\
\text { Switzerland }\end{array}$ & $\begin{array}{l}\text { Philippe Frank } \\
\text { USA }\end{array}$ & $\begin{array}{l}\text { Yusuke Furukawa } \\
\text { Japan }\end{array}$ \\
\hline $\begin{array}{l}\text { Gary Firestone } \\
\text { USA }\end{array}$ & $\begin{array}{l}\text { Callum Fraser } \\
\text { UK }\end{array}$ & $\begin{array}{l}\text { Junji Furuse } \\
\text { Japan }\end{array}$ \\
\hline $\begin{array}{l}\text { Jodie M Fleming } \\
\text { USA }\end{array}$ & $\begin{array}{l}\text { Jonna Frasor } \\
\text { USA }\end{array}$ & $\begin{array}{l}\text { Koh Furuta } \\
\text { Japan }\end{array}$ \\
\hline $\begin{array}{l}\text { Emmy Fleuren } \\
\text { Netherlands }\end{array}$ & $\begin{array}{l}\text { Alex Freeman } \\
\text { UK }\end{array}$ & $\begin{array}{l}\text { Mayuko Furuta } \\
\text { Japan }\end{array}$ \\
\hline $\begin{array}{l}\text { Mirembe Florenc } \\
\text { Uganda }\end{array}$ & $\begin{array}{l}\text { Manuel Fresno } \\
\text { Spain }\end{array}$ & $\begin{array}{l}\text { Hideki Furuya } \\
\text { USA }\end{array}$ \\
\hline $\begin{array}{l}\text { Tullio Florio } \\
\text { Italy }\end{array}$ & $\begin{array}{l}\text { Daniela Frezzetti } \\
\text { Italy }\end{array}$ & $\begin{array}{l}\text { Martin Götte } \\
\text { Germany }\end{array}$ \\
\hline
\end{tabular}




\begin{tabular}{|c|c|c|}
\hline $\begin{array}{l}\text { Shiva Keshava Gaddam B } \\
\text { USA }\end{array}$ & $\begin{array}{l}\text { José Manuel García-Heredia } \\
\text { Spain }\end{array}$ & $\begin{array}{l}\text { Farid Ghadessy } \\
\text { Singapore }\end{array}$ \\
\hline $\begin{array}{l}\text { Jochen Gaedcke } \\
\text { Germany }\end{array}$ & $\begin{array}{l}\text { Ramón García-Sanz } \\
\text { Spain }\end{array}$ & $\begin{array}{l}\text { Hazem Ghebeh Saudi } \\
\text { Arabia }\end{array}$ \\
\hline $\begin{array}{l}\text { Bertino Gaetano } \\
\text { Italy }\end{array}$ & $\begin{array}{l}\text { Paul Gardner } \\
\text { USA }\end{array}$ & $\begin{array}{l}\text { Paola Ghiorzo } \\
\text { Italy }\end{array}$ \\
\hline $\begin{array}{l}\text { Ajeet Gajra } \\
\text { USA }\end{array}$ & $\begin{array}{l}\text { David Garfield } \\
\text { China }\end{array}$ & $\begin{array}{l}\text { Paramita Ghosh } \\
\text { USA }\end{array}$ \\
\hline $\begin{array}{l}\text { Umberto Galderisi } \\
\text { Italy }\end{array}$ & $\begin{array}{l}\text { Manuela Gariboldi } \\
\text { Italy }\end{array}$ & $\begin{array}{l}\text { Goutam Ghosh Choudhury } \\
\text { USA }\end{array}$ \\
\hline $\begin{array}{l}\text { Miguel Gallego } \\
\text { Spain }\end{array}$ & $\begin{array}{l}\text { Scott Garrett } \\
\text { USA }\end{array}$ & $\begin{array}{l}\text { Juliana Giacomazzi } \\
\text { Brazil }\end{array}$ \\
\hline $\begin{array}{l}\text { Brenda Gallie } \\
\text { Canada }\end{array}$ & $\begin{array}{l}\text { Gasim Gasim } \\
\text { Sudan }\end{array}$ & $\begin{array}{l}\text { Vincenzo Giancotti } \\
\text { Italy }\end{array}$ \\
\hline $\begin{array}{l}\text { Valerio Gallotta } \\
\text { Italy }\end{array}$ & $\begin{array}{l}\text { Pierluigi Gasparini } \\
\text { USA }\end{array}$ & $\begin{array}{l}\text { Elisa Giannoni } \\
\text { Italy }\end{array}$ \\
\hline $\begin{array}{l}\text { Paolo Gandellini } \\
\text { Italy }\end{array}$ & $\begin{array}{l}\text { Mia Gaudet } \\
\text { USA }\end{array}$ & $\begin{array}{l}\text { Bruce Giantonio } \\
\text { USA }\end{array}$ \\
\hline $\begin{array}{l}\text { Ripal Gandhi } \\
\text { USA }\end{array}$ & $\begin{array}{l}\text { Luke Gaughan } \\
\text { UK }\end{array}$ & $\begin{array}{l}\text { Spencer Gibson } \\
\text { Canada }\end{array}$ \\
\hline $\begin{array}{l}\text { Sara Gandini } \\
\text { Italy }\end{array}$ & $\begin{array}{l}\text { Julie Gavard } \\
\text { France }\end{array}$ & $\begin{array}{l}\text { Roben Gieling } \\
\text { UK }\end{array}$ \\
\hline $\begin{array}{l}\text { Wei-Qiang Gao } \\
\text { China }\end{array}$ & $\begin{array}{l}\text { Maria Gavriatopoulou } \\
\text { Greece }\end{array}$ & $\begin{array}{l}\text { Gretchen Gierach } \\
\text { USA }\end{array}$ \\
\hline $\begin{array}{l}\text { Ai Gao } \\
\text { China }\end{array}$ & $\begin{array}{l}\text { Hiram Gay } \\
\text { USA }\end{array}$ & $\begin{array}{l}\text { Carolina Gigek } \\
\text { Brazil }\end{array}$ \\
\hline $\begin{array}{l}\text { Chunfang Gao } \\
\text { China }\end{array}$ & $\begin{array}{l}\text { Bishoy Gayed } \\
\text { USA }\end{array}$ & $\begin{array}{l}\text { Ignacio Gil-Bazo } \\
\text { Spain }\end{array}$ \\
\hline $\begin{array}{l}\text { Qinyan Gao } \\
\text { China }\end{array}$ & $\begin{array}{l}\text { Christopher Gayer } \\
\text { USA }\end{array}$ & $\begin{array}{l}\text { Ethel Gilbert } \\
\text { USA }\end{array}$ \\
\hline $\begin{array}{l}\text { Paul Gao } \\
\text { USA }\end{array}$ & $\begin{array}{l}\text { Akihiko Gemma } \\
\text { Japan }\end{array}$ & $\begin{array}{l}\text { Blake Gilks } \\
\text { Canada }\end{array}$ \\
\hline $\begin{array}{l}\text { Haidong Gao } \\
\text { China }\end{array}$ & $\begin{array}{l}\text { Domenico Genovesi } \\
\text { Italy }\end{array}$ & $\begin{array}{l}\text { Jerome Gilleron } \\
\text { Germany }\end{array}$ \\
\hline $\begin{array}{l}\text { Wen Gao } \\
\text { China }\end{array}$ & $\begin{array}{l}\text { Alex Georgakilas } \\
\text { Greece }\end{array}$ & $\begin{array}{l}\text { Bernhard Gillissen } \\
\text { Germany }\end{array}$ \\
\hline $\begin{array}{l}\text { Claus Garbe } \\
\text { Germany }\end{array}$ & $\begin{array}{l}\text { Vassilis Georgoulias } \\
\text { Greece }\end{array}$ & $\begin{array}{l}\text { Etel Gimba } \\
\text { Brazil }\end{array}$ \\
\hline $\begin{array}{l}\text { Spiros D. Garbis } \\
\text { UK }\end{array}$ & $\begin{array}{l}\text { Holger Gerullis } \\
\text { Germany }\end{array}$ & $\begin{array}{l}\text { Antonio Z Gimeno García } \\
\text { Spain }\end{array}$ \\
\hline $\begin{array}{l}\text { Juan F Garcia } \\
\text { Spain }\end{array}$ & $\begin{array}{l}\text { Robert Getzenberg } \\
\text { USA }\end{array}$ & $\begin{array}{l}\text { Doron Ginsberg } \\
\text { Israel }\end{array}$ \\
\hline $\begin{array}{l}\text { Montse Garcia } \\
\text { Spain }\end{array}$ & $\begin{array}{l}\text { Sara Ghaderi } \\
\text { Norway }\end{array}$ & $\begin{array}{l}\text { Rosaria Giordano } \\
\text { Italy }\end{array}$ \\
\hline
\end{tabular}


Paolo Giorgi Rossi

Italy

Elisa Giovannetti

Netherlands

Albert Girotti
USA
David Gius
USA
Babak Givi
USA
Simona Glasberg
Israel

Israel

David Glass

USA

Sacha Gnjatic

USA

Roseline Godbout

Canada

James Goedert

USA

James Going

UK

Vita Golubovskaya

USA

Carolina Gomes

Brazil

Dawidson Gomes

Brazil

Jorge Gomez

USA

Isabel Gomez Monterrey

Italy

Anne Gompel

France

Elena Goncharova

USA

Zhaohui Gong

China

Céline Gongora

France

Lou Gonsalves

USA

Maria Cristina Gonzalez

Brazil
Patrick Gonzalez

France

Patrick Goodman

Ireland

Satish Gopal

Malawi

Priya Gopalan

USA

Gregor Gorkiewicz

Austria

Rei Goto

Japan

Michael Gottesman

USA

Ioannis Gounaris

UK

Erin Grady

USA

Ullrich Graeven

Germany

Maria Gramatges

USA

Diana Greenfield

UK

Darren C Greenwood

UK

Peter Greer

Canada

Christopher Griffith

USA

Thomas Griffith

USA

Giovanni Grignani

Italy

Anita Grigoriadis

UK

Christoph Grimm

Austria

Chloe Grimmett

UK

Peter Grimminger

Germany

Fabio Grizzi

Italy
Christian Grohe

Germany

Francesco Grossi

Italy

Kenneth Grossmann

USA

Beth Grunfeld

UK

Eva Grunfeld

Canada

Viktor Grünwald

Germany

Guoli Gu

China

Yajun Gu

China

Xiaoxiang Guan

China

Yongsong Guan

China

Nathalie Guedj

France

Arndt Guentsch

Germany

Vince Guerriero

USA

Jih-Hwa Guh

Taiwan

Massimo Guidoboni

Italy

Maria Valle Guijarro

USA

Andre Guimaraes

Brazil

Margaret Gulley

USA

Mehmet Gunduz

Japan

Deliang Guo

USA

Wei Guo

China

Li Guo

China 
Wei-Jian Guo

China

Xiaozhong Guo

China

Zong Sheng Guo

USA

Junming Guo

China

Digant Gupta

USA

Nalini Gupta

India

Phalguni Gupta

India

Marianne Guren

Norway

Kurt Gust

USA

Dmitriy Gutkin

USA

Mina Ha

South Korea

Ulrich Hacker

Germany

Christina Hackl

Germany

Ahmed Haddad

USA

Peyman Hadji

Germany

Costas Hadjipanayis

USA

Andreas Hadjisavvas

Cyprus

Anne Irene Hagen

Norway

Ki-Baik Hahm

South Korea

Benjamin Haibe-Kains

Canada

Jörg Haier

Germany

Hidde Haisma

Netherlands
A Ari Hakimi

USA

Richard Halberg

USA

Michael Halpern

USA

Gerhard Hamilton

Austria

William Hamilton

UK

Bruce Hammock

USA

Weidong Han

China

Wenling Han

China

Henry Han

USA

Yong Han

China

Rachel Hanisch

France

Matthew Hankins

UK

Torben Hansen

Denmark

Dapeng Hao

Macao

Jihui Hao

China

Sheng-Po Hao

Taiwan

Hisashi Harada

USA

Jennifer Hardingham

Australia

James Hardwick

Netherlands

Ljubica Harhaji

Serbia

Valsala Haridas

USA

J. Chuck Harrell

USA
Holly Harris

USA

Steven Hart

USA

Lowell Hart

USA

Jaana Hartikainen

Finland

John Hartley

UK

Sheri Hartman

USA

Sayed Hashemi

Netherlands

Naozumi Hashimoto

Japan

Noor Hasima

Malaysia

Brian Hasinoff

Canada

Ursula Hasler-Strub

Switzerland

Cesare Hassan

Italy

Raffit Hassan

USA

Brad Haverkos

USA

Lukas J.A.C. Hawinkels

Netherlands

David Hawkins

USA

Sandi Hayes

Australia

John Hays

USA

Aiwu He

USA

Hong He

Australia

Jin He

USA

Qing He

China 


\begin{tabular}{|c|c|c|}
\hline $\begin{array}{l}\text { Shannon Healy } \\
\text { Canada }\end{array}$ & $\begin{array}{l}\text { Aram Hezel } \\
\text { USA }\end{array}$ & $\begin{array}{l}\text { Thomas Hofmann } \\
\text { Germany }\end{array}$ \\
\hline $\begin{array}{l}\text { Lionel Hebbard } \\
\text { Australia }\end{array}$ & $\begin{array}{l}\text { Toyoaki Hida } \\
\text { Japan }\end{array}$ & $\begin{array}{l}\text { Peter Hollenhorst } \\
\text { USA }\end{array}$ \\
\hline $\begin{array}{l}\text { Ingrid Hedenfalk } \\
\text { Sweden }\end{array}$ & $\begin{array}{l}\text { Jin-Ichi Hida } \\
\text { Japan }\end{array}$ & $\begin{array}{l}\text { Antoinette Hollestelle } \\
\text { Netherlands }\end{array}$ \\
\hline $\begin{array}{l}\text { Steffen Heeg } \\
\text { Germany }\end{array}$ & $\begin{array}{l}\text { Lotte Hiddingh } \\
\text { Netherlands }\end{array}$ & $\begin{array}{l}\text { Lars Holmberg } \\
\text { UK }\end{array}$ \\
\hline $\begin{array}{l}\text { Christopher Heeschen } \\
\text { Spain }\end{array}$ & $\begin{array}{l}\text { Geoff Higgins } \\
\text { UK }\end{array}$ & $\begin{array}{l}\text { Dennis Holmes } \\
\text { USA }\end{array}$ \\
\hline $\begin{array}{l}\text { Balazs Hegedus } \\
\text { Austria }\end{array}$ & $\begin{array}{l}\text { Mitsunori Higuchi } \\
\text { Japan }\end{array}$ & $\begin{array}{l}\text { Jeff Holst } \\
\text { Australia }\end{array}$ \\
\hline $\begin{array}{l}\text { Axel Hegele } \\
\text { Germany }\end{array}$ & $\begin{array}{l}\text { Masahiro Higuchi } \\
\text { USA }\end{array}$ & $\begin{array}{l}\text { Santosh Honavar } \\
\text { India }\end{array}$ \\
\hline $\begin{array}{l}\text { Joost Hegmans } \\
\text { Netherlands }\end{array}$ & $\begin{array}{l}\text { Frans Hilgers } \\
\text { Netherlands }\end{array}$ & $\begin{array}{l}\text { Kazuo Honda } \\
\text { Japan }\end{array}$ \\
\hline $\begin{array}{l}\text { Tuomas Heikkinen } \\
\text { Finland }\end{array}$ & $\begin{array}{l}\text { Andrew Hill } \\
\text { New Zealand }\end{array}$ & $\begin{array}{l}\text { Angela Hong } \\
\text { Australia }\end{array}$ \\
\hline $\begin{array}{l}\text { Max Heiland } \\
\text { Germany }\end{array}$ & $\begin{array}{l}\text { Uwe Himmelreich } \\
\text { Belgium }\end{array}$ & $\begin{array}{l}\text { Chi-Chen Hong } \\
\text { USA }\end{array}$ \\
\hline $\begin{array}{l}\text { Elmar Heinrich } \\
\text { Germany }\end{array}$ & $\begin{array}{l}\text { Joe Hines } \\
\text { USA }\end{array}$ & $\begin{array}{l}\text { Yong Sang Hong } \\
\text { South Korea }\end{array}$ \\
\hline $\begin{array}{l}\text { Florian Heitz } \\
\text { Germany }\end{array}$ & $\begin{array}{l}\text { Okio Hino } \\
\text { Japan }\end{array}$ & $\begin{array}{l}\text { Kimi Honma } \\
\text { Australia }\end{array}$ \\
\hline $\begin{array}{l}\text { Ellen Heitzer } \\
\text { Austria }\end{array}$ & $\begin{array}{l}\text { Christoph Hirche } \\
\text { Germany }\end{array}$ & $\begin{array}{l}\text { Naoko Honma } \\
\text { Japan }\end{array}$ \\
\hline $\begin{array}{l}\text { Eric Henderson } \\
\text { USA }\end{array}$ & $\begin{array}{l}\text { Seiichi Hirota } \\
\text { Japan }\end{array}$ & $\begin{array}{l}\text { Craig Horbinski } \\
\text { USA }\end{array}$ \\
\hline $\begin{array}{l}\text { Mary Hendrix } \\
\text { USA }\end{array}$ & $\begin{array}{l}\text { Bradford Hirsch } \\
\text { USA }\end{array}$ & $\begin{array}{l}\text { Ryouichi Horie } \\
\text { Japan }\end{array}$ \\
\hline $\begin{array}{l}\text { Charles Hennemeyer } \\
\text { USA }\end{array}$ & $\begin{array}{l}\text { Hal Hirte } \\
\text { Canada }\end{array}$ & $\begin{array}{l}\text { Hidehito Horinouchi } \\
\text { Japan }\end{array}$ \\
\hline $\begin{array}{l}\text { John Henson } \\
\text { USA }\end{array}$ & $\begin{array}{l}\text { Asahi Hishida } \\
\text { Japan }\end{array}$ & $\begin{array}{l}\text { Martinho Horta } \\
\text { Brazil }\end{array}$ \\
\hline $\begin{array}{l}\text { Javier Hernandez-Losa } \\
\text { Spain }\end{array}$ & $\begin{array}{l}\text { Taro Hitosugi } \\
\text { USA }\end{array}$ & $\begin{array}{l}\text { H Dean Hosgood } \\
\text { USA }\end{array}$ \\
\hline $\begin{array}{l}\text { Shawn Hervey-Jumper } \\
\text { USA }\end{array}$ & $\begin{array}{l}\text { Steven Hochwald } \\
\text { USA }\end{array}$ & $\begin{array}{l}\text { Isamu Hoshino } \\
\text { Japan }\end{array}$ \\
\hline $\begin{array}{l}\text { Marcin Hetnal } \\
\text { Poland }\end{array}$ & $\begin{array}{l}\text { Robert Hoffman } \\
\text { USA }\end{array}$ & $\begin{array}{l}\text { Katsuyuki Hotta } \\
\text { Japan }\end{array}$ \\
\hline $\begin{array}{l}\text { Michael Heuser } \\
\text { Germany }\end{array}$ & $\begin{array}{l}\text { Michael Hoffmeister } \\
\text { Germany }\end{array}$ & $\begin{array}{l}\text { Scott Howard } \\
\text { USA }\end{array}$ \\
\hline $\begin{array}{l}\text { Holger Heyn } \\
\text { Spain }\end{array}$ & $\begin{array}{l}\text { Ilse Hofmann } \\
\text { Germany }\end{array}$ & $\begin{array}{l}\text { Debra Howell } \\
\text { UK }\end{array}$ \\
\hline
\end{tabular}


Viive Howell

Australia

Morten Høyer

Denmark

Jenn-Ren Hsiao

Taiwan

Michael Hsiao

Taiwan

Chiun Hsu

Taiwan

Cheng-Lung Hsu

Taiwan

Brian $\mathrm{Hu}$

USA

Chaosu Hu

China

Yi Hu

China

Xi Chun Hu

China

Hsuan-Ying Huang

Taiwan

Cai Huang

USA

Chih-Jen Huang

Taiwan

Chi-Cheng Huang

Taiwan

Chao-Cheng Huang

Taiwan

Eng-Yen Huang

Taiwan

Wen-Yi Huang

USA

Ying Huang

USA

Yi Huang

USA

Zhaohui Huang

China

Weihuang Huang

China

Paul Huang

UK
Michael Hubalek

Austria

Peter Huber

Germany

John Hudson

Canada

David Hughes

USA

Jooryung Huh

South Korea

Stefanie Huhn

Germany

David Hui

USA

Zhouguang Hui

China

Luo Huiyan

China

Michael Hundemer

Germany

Erik Huntzicker

USA

Sara Hurvitz

USA

Jayne Hutchinson

UK

Ji-Young Hwang

South Korea

Tae-Ho Hwang

South Korea

Elias Hyams

USA

Per Hydbring

USA

David Hyman

USA

Nancy Hynes

Switzerland

Ichinosuke Hyodo

Japan

Roberto Iacovelli

Italy

Merdol Ibrahim

UK
Wataru Ichikawa

Japan

Kenneth Iczkowski

USA

Satoshi Igawa

Japan

Michaela Ihle

Germany

Hiroshi Ikai

Japan

Junichiro Ikeda

Japan

Masahide Ikeguchi

Japan

Carolina Ilkow

Canada

Martin Illemann

Denmark

Mohammad Ilyas

UK

Kazushi Imai

Japan

Takeshi Imamura

Japan

Stefano Indraccolo

Italy

Maurizio V Infante

Italy

Robert Ingham

Canada

Pasquale Innominato

France

Hidenori Inohara

Japan

Akira Inoue

Japan

Paul Insel

USA

Marisa Ionta

Brazil

Marilena Iorio

Italy

Sarosh Irani

UK 


\begin{tabular}{|c|c|c|}
\hline Elena Irollo & Marko Jakopovic & Collene Jeter \\
\hline Germany & Croatia & USA \\
\hline Claudio Isella & Sirpa Jalkanen & Bruno Jham \\
\hline Italy & Finland & USA \\
\hline Ciro Isidoro & Kaiser Jamil & Guixiang Ji \\
\hline Italy & India & China \\
\hline Nina Isoherranen & Sabina Janciauskiene & Xin-Ying Ji \\
\hline USA & Sweden & China \\
\hline Yasuhiro Ito & Tae Jung Jang & Weiping Jia \\
\hline Japan & South Korea & China \\
\hline Yuri Ito & Judith Jans & Wei Jiang \\
\hline Japan & Netherlands & China \\
\hline Ken Itoh & Emiel Janssen & Min Jiang \\
\hline Japan & Norway & China \\
\hline Motoki Iwasaki & Klaus-Peter Janssen & Wen Jiang \\
\hline Japan & Germany & USA \\
\hline Yoshimi Iwasaki & Thierry Jarde & Shih Sheng Jiang \\
\hline Japan & Australia & Taiwan \\
\hline Tomoko Iwata & Mona Jeffreys & Tun Jie \\
\hline UK & UK & USA \\
\hline Obiajulu Iwenofu & Valerie Jenkins & Tang Jie \\
\hline USA & UK & China \\
\hline N Gopalakrishna Iyer & Jørgen Bjerggaard Jensen & Camilo Jimenez \\
\hline Singapore & Denmark & USA \\
\hline Jakob Izbicki & Roxanne Jensen & Rodrigo Jimenez Garcia \\
\hline Germany & USA & Spain \\
\hline Evgeny Izumchenko & Hae Myung Jeon & Haifeng Jin \\
\hline USA & South Korea & China \\
\hline Maria Jackson & Seong-Yun Jeong & Shouguang Jin \\
\hline Jamaica & South Korea & USA \\
\hline Anne-Birgitte Jacobsen & Branislav Jeremic & Young Woo Jin \\
\hline Norway & Serbia & South Korea \\
\hline Judith S Jacobson & Fredrik Jerhammar & Chunxia Jing \\
\hline USA & Sweden & China \\
\hline Marie-Lise Jaffrain-Rea & Carmen Jeronimo & Young Suk Jo \\
\hline Italy & Portugal & South Korea \\
\hline Harriët Jager-Wittenaar & Udo Jeschke & Aart G Jochemsen \\
\hline Netherlands & Germany & Netherlands \\
\hline Preetesh Jain & Mahsa Jessri & Karin Joehrer \\
\hline USA & Canada & Austria \\
\hline Praveen Jaiswal & John Jessup & Markus Joerger \\
\hline India & USA & Switzerland \\
\hline Jens Jakob & Hernandez Jesus $M$ & David Johnson \\
\hline Germany & Spain & USA \\
\hline
\end{tabular}


Matthew Johnson

USA

Theron Johnson

Germany

Mickaila Johnston

USA

V. Craig Jordan

USA

Bharat Joshi

USA

Elmar Joura

Austria

Alicja Jozkowicz

Poland

Manfred Jücker

Germany

Ian Judson

UK

Bettina Julin

Sweden

Andreas Jung

Germany

Young Jung

South Korea

Kerstin Junker

Germany

Therese Juul

Denmark

Guillermo Juvenal

Argentina

Hayyam Kiratli

Turkey

Lars Kaderali

Germany

Pinar Kadioglu

Turkey

Shigenori Kadowaki

Japan

Keita Kai

Japan

Sham Kakar

USA

Kiran Kakarala

USA
John Kalbfleisch

USA

Vijaykumar Kale

USA

Kevin Kalinsky

USA

Enikoe Kallay

Austria

Galatea Kallergi

Greece

Raghu Kalluri

USA

Charis Kalogirou

Germany

Christina Kalpadakis

Greece

Arif Kamal

USA

Bozena Kaminska

Poland

Izumi Kamiya

Japan

Ellen Kampman

Netherlands

Ghodsieh Kamrani

Iran

Ryuichi Kanai

Japan

Kent Kanao

Japan

Mustapha Kandouz

USA

Kazunori Kanehira

USA

Peggy Kanellou

Greece

Jung Hun Kang

South Korea

Stephan Kanzler

Germany

Jessica Kao

USA

Matthias Kappler

Germany
Georgios Karagkounis

USA

Amer Karam

USA

Jose Karam

USA

Anna Karlsson

Sweden

Rotem Karni

Israel

Radha Krishna Murthy Karuturi

Singapore

Takahiro Kasamatsu

Japan

Stefan Kasper

Germany

Wassim Kassouf

Canada

Kota Katanoda

Japan

Katsuaki Kato

Japan

Masaru Katoh

Japan

Rachel Katzenellenbogen

USA

Rondi Kauffmann

USA

Ruchika Kaul-Ghanekar

India

Koji Kawakami

Japan

Chul-Seung Kay

South Korea

Youqiang Ke

UK

Bhumsuk Keam

South Korea

Ulrich Keilholz

Germany

Linda Kelemen

Canada

Kimberly Kelly

USA 
Patrick Kelly

USA

Stefan Kempa

Germany

Paraic Kenny

USA

Lucyna Kepka

Poland

Ruth Keri

USA

Michael Kershaw

Australia

Santosh Kesari

USA

Aparna Kesarwala

USA

Dineo Khabele

USA

Hadi Khan

USA

Kum Kum Khanna

Australia

\section{Adnan Khattak}

Australia

Seong Lin Khaw

Australia

Surapan Khunamornpong

Thailand

Tobias Kiesslich

Austria

Thomas Kietzmann

Finland

Kotaro Kiga

Japan

Sarah Kiguli

Uganda

Eiji Kikuchi

Japan

Elaine Kilgour

UK

Hyoung-Ryoul Kim

South Korea

Eugene Kim

USA
Hyung-Ho Kim

South Korea

Jung-Ae Kim

South Korea

James Kim

USA

Joseph Kim

USA

Betty Y.S. Kim

USA

Su Woon Kim

Singapore

Sung Joo Kim

South Korea

Marianne Kim

USA

Sun Jung Kim

South Korea

Tae Won Kim

South Korea

Won Gu Kim

South Korea

Young Wan Kim

South Korea

T Peter Kingham

USA

Iva Kirac

Croatia

Jutta Kirfel

Germany

Hiroyuki Kirikoshi

Japan

Yohei Kirino

USA

Antti Kivelä

Finland

Kristina Kjaerheim

Norway

Wolfram Klapper

Germany

Giseli Klassen

Brazil

Christoph Klein

Germany
Martin Klein

Netherlands

Robert Klein

USA

Miriam Kleiter

Austria

Samuel Klempner

USA

Dennis Klinman

USA

Roman Kloeckner

Germany

Petra Klose

Germany

Olaf Klungel

Netherlands

Stian Knappskog

Norway

Michael Knauer

Switzerland

Steen Knudsen

Denmark

Ben Ko

Hong Kong

Jiunn-Liang Ko

Taiwan

Michiya Kobayashi

Japan

Wayne Koch

USA

Hemant Kocher

UK

Martin Koebel

Canada

Devin Koestler

USA

Barbara Kofler

Austria

Fumitaka Koga

Japan

Takahiko Kogai

Japan

Yasuhiro Koh

Japan 
Takashi Kohno

Japan

Yasuo Kokai

Japan

Anna Kolovou

Greece

Hiroshi Kondoh

Japan

Konstantinos Konstantopoulos

USA

Christos Kontos

Greece

Despina Kontos

USA

Rituraj Konwar

India

József Kónya

Hungary

Bon-Kyoung Koo

UK

Dong Hoe Koo

South Korea

Joseph Koopmeiners

USA

Marta Korbonits

Uruguay

Murray Korc

USA

Hasan Korkaya

USA

Penelope Korkolopoulou

Greece

Masafumi Koshiyama

Japan

Zsofia Kote-Jarai

UK

Madhuri Koti

Canada

Ashwin Kotnis

India

Svetlana Kotova

USA

Athanasios Kotsakis

Greece
Athanassios Kotsinas

Greece

Vassilis Kouloulias

Greece

Zaklina Kovacevic

Australia

Gyoergy Kovacs

Germany

Nobuyuki Koyama

Japan

Sergei Kozlov

Australia

Shingo Kozono

Japan

Dominique Kranz

Germany

Pawel Krawczyk

Poland

Pavel Krejci

Czech Republic

Marina Kreutz

Germany

Samaya Krishnan

USA

Lasse Sommer Kristensen

Denmark

Stephan Kruck

Germany

Wlodzimierz Krzyzosiak

Poland

Rui Kuang

USA

Lisha Kuang

USA

Lucia Kucerova

Slovakia

Veerapol Kukongviriyapan

Thailand

Teru Kumagi

Japan

Ashok Kumar

USA

Shiyam Kumar

Oman
Prince Kumar

India

Ranjit Kumar

USA

Gopal Kundu

India

Hsiu-Ni Kung

Taiwan

Etsuo Kunieda

Japan

Joachim Kunz

Germany

Catarina Alisa Kunze

Germany

Jolanta Kupryjanczyk

Poland

Kentaro Kurashina

Japan

Masafumi Kurosumi

Japan

Boris Kuvshinoff

USA

Hiroyuki Kuwano

Japan

Marilyn Kwan

USA

Ioannis Kyvernitakis

Germany

Simon Laban

Germany

Chann Lagadec

France

Andrei Lagaru

UK

Catherine Lai

USA

Lily Lai

USA

Barry Laird

UK

Lisenn Lalier

France

Vaia Lambadiari

Greece 
Daniel Lambert

UK

Nadia Lampiasi

Italy

Jeffrey Landercasper

USA

Deanna Lane

USA

Kerstin Lang

Germany

Sven Lang

Germany

Rupert Langer

Switzerland

Cord Langner

Austria

Andrea Lania

Italy

Helena Länsimies-Antikainen

Finland

Constantin Lapa

Germany

Michael Larkin

UK

Mette Bach Larsen

Denmark

Christer Larsson

Sweden

Ahmed Lasfar

USA

Ulrik Lassen

Denmark

Justin Lathia

USA

David Latini

USA

Joseph W.Y. Lau

Hong Kong

Darryl Lau

USA

Vincent Launay-Vacher

France

Matthias Lauth

Germany
Nicole Lavender

USA

Yuri Lazebnik

USA

Lu Le

USA

Avi Leader

Israel

Sophie Lebel

Canada

Arier Lee

New Zealand

Cheng Chi Lee

USA

Cheng-Han Lee

Canada

Chien-Hung Lee

Taiwan

Hsin-Chen Lee

Taiwan

Ching-Chih Lee

Taiwan

Han-Woong Lee

South Korea

Hyeon-Woo Lee

South Korea

Jae Lyun Lee

South Korea

Jung-Il Lee

South Korea

Ju-Seog Lee

USA

Jehn Chuan Lee

Taiwan

Hwajeong Lee

USA

Jung-Min Lee

USA

Kin Chung Lee

Hong Kong

Se-Hoon Lee

South Korea

Simon J. Craddock Lee

USA
Kin Wah Lee

Hong Kong

Wei-Hwa Lee

Taiwan

Qunying Lei

China

Mario Leitao Jr

USA

Catherine Lejeune

France

Sophie Lelievre

USA

Wei-Dong Leng

China

Claudia Lengerke

Switzerland

Jochen Lennerz

USA

Ryan Lennon

USA

Dido Lenze

Germany

Francesco Leone

Italy

Derek Leroith

USA

Gregory Lesinski

USA

Eleonora Leucci

Belgium

Anna Leung

USA

David Levens

USA

Francis Levi

France

Baoqing Li

USA

Chunyu Li

China

Jin Li

China

Huabing Li

USA 
Jingmei Li

Singapore

Shau-Hsuan Li

Taiwan

Wei Li

USA

Hongyan Li

USA

Jinjun Li

China

Long-Cheng Li

USA

Shao-Qiang Li

China

Xiaobo Li

China

Jianming Li

China

Jun Li

China

Long Shan Li

USA

Madeline Li

Canada

Qiang Li

USA

Rui Li

China

Wen Li

China

Ya-Hsin Li

Taiwan

Zaibo Li

USA

Min Lian

USA

Evi Lianidou

Greece

Yung-Po Liaw

Taiwan

Massimo Libra

Italy

Karen Liby

USA
Jonathan Licht

USA

David Liebner

USA

Sandra Liekens

Belgium

Jong-Baeck Lim

South Korea

Carmen Lima

Brazil

Carlos A. Lima

Brazil

Chih-Lung Lin

Taiwan

Ho-Sheng Lin

USA

Huey-Jen Lin

USA

Jiumao Lin

China

Kenneth Lin

USA

Kwang-Huei Lin

Taiwan

Yu-Sheng Lin

USA

Li Lin

China

Lijuan Lin

China

Wei-Che Lin

Taiwan

Xun Lin

USA

Zhenhua Lin

China

Hannah Linden

USA

Chen Ling

USA

Zhi-Qiang Ling

China

Isaac Lipkus

USA
Baorui Liu

China

Hong Liu

USA

Delong Liu

USA

Ji-Bin Liu

USA

Lifang Liu

Netherlands

Houbao Liu

China

Tianshu Liu

China

Bo Liu

China

Jianming Liu

Cocos Keeling Islands

Zuqiang Liu

USA

Shing-Hwa Liu

Taiwan

Tao Liu

Australia

Weiguo Liu

USA

Xiangguo Liu

China

Xiaochun Liu

USA

Yan Liu

USA

Zhili Liu

China

Zhensheng Liu

USA

Gema Llort

Spain

Bryan Lo

Canada

Simon Lo

USA

Filippo Lococo

Italy 
Ines Lohse

Canada

Niklas Loman

Sweden

Giuseppe Lombardi

Italy

Fulvio Lonardo

USA

Cheryl London

USA

Kara Long Roche

USA

Adhemar Longatto Filho

Portugal

Luca Longo

Italy

Elena Lopez

Spain

Gonzalo Lopez

USA

Jose Antonio Lopez-Guerrero

Spain

Francisco Lopez-Hernandez

Spain

Ian Lorimer

Canada

Attila Lorincz

UK

Joannie Lortet-Tieulent

USA

Corina Lorz

Spain

Kevin R. Loughlin

USA

Laercio Gomes Lourenco

Brazil

Aoife Lowery

Ireland

Hua Lu

USA

Kai-Hsi Lu

Taiwan

Shen Lu

China
Xiaomei Lu

China

Weiyue Lu

China

Sudjit Luanpitpong

USA

Giovanni Lughezzani

Italy

Alessandro Lugli

Switzerland

Eiliv Lund

Norway

Kenneth Lundstrom

Switzerland

Pengcheng Luo

China

Sheng-Dean Luo

Taiwan

Shuhong Luo

USA

Yunus Luqmani

Kuwait

John Lurain

USA

Rodney Luwor

Australia

Yi Lv

China

Herbert Lyerly

USA

Courtney Lyles

USA

Marita Lynagh

Australia

Heidi Lyng

Norway

Hong Ma

China

Chenming Ma

Germany

Daoxin Ma

China

Jun Ma

China
Lin Ma

China

Qingyong Ma

China

Bruce Macher

USA

Gerardo Mackenzie

USA

Angeliki Magklara

Greece

Kelly Magliocca

USA

Jamal Mahajna

Israel

Marthandan Mahalingam

USA

Umesh Mahanshetty

India

Reza Mahdavi

Iran

Georg Mahlknecht

Israel

Mamun Mahtab

Bangladesh

Hai-Qiang Mai

China

Shishir Maithel

USA

Shahana Majid

USA

Keivan Majidzadeh-A

Iran

Carl Maki

USA

Paolo Malatesta

Italy

Núria Malats

Spain

Jitka Malcikova

Czech Republic

Peter Malfertheiner

Germany

Eirik Malinen

Norway 


\begin{tabular}{|c|c|c|}
\hline $\begin{array}{l}\text { Sandra Mallone } \\
\text { Italy }\end{array}$ & $\begin{array}{l}\text { Caterina Marchiò } \\
\text { Italy }\end{array}$ & $\begin{array}{l}\text { Floriana Mascilini } \\
\text { Italy }\end{array}$ \\
\hline $\begin{array}{l}\text { Joshua Mammen } \\
\text { USA }\end{array}$ & $\begin{array}{l}\text { Raphaël Maréchal } \\
\text { Belgium }\end{array}$ & $\begin{array}{l}\text { Liliane Massade } \\
\text { France }\end{array}$ \\
\hline $\begin{array}{l}\text { Aaron Mammoser } \\
\text { USA }\end{array}$ & $\begin{array}{l}\text { Johanna Maree } \\
\text { South Africa }\end{array}$ & $\begin{array}{l}\text { Andrew Massey } \\
\text { UK }\end{array}$ \\
\hline $\begin{array}{l}\text { Kwan Man } \\
\text { China }\end{array}$ & $\begin{array}{l}\text { Julie Margenthaler } \\
\text { USA }\end{array}$ & $\begin{array}{l}\text { Norikazu Masuda } \\
\text { Japan }\end{array}$ \\
\hline $\begin{array}{l}\text { Parmeet Manchanda } \\
\text { USA }\end{array}$ & $\begin{array}{l}\text { Boris Margulis Russian } \\
\text { Federation }\end{array}$ & $\begin{array}{l}\text { María-Victoria Mateos } \\
\text { Spain }\end{array}$ \\
\hline $\begin{array}{l}\text { Julien Mancini } \\
\text { France }\end{array}$ & $\begin{array}{l}\text { Vitaly Margulis } \\
\text { USA }\end{array}$ & $\begin{array}{l}\text { Augusto Matos } \\
\text { Portugal }\end{array}$ \\
\hline $\begin{array}{l}\text { Chandi Mandal } \\
\text { India }\end{array}$ & $\begin{array}{l}\text { Isabelle Maridonneau-Parini } \\
\text { France }\end{array}$ & $\begin{array}{l}\text { Ayako Matsuda } \\
\text { Japan }\end{array}$ \\
\hline $\begin{array}{l}\text { Syamsundar Mandal } \\
\text { India }\end{array}$ & $\begin{array}{l}\text { Jose Marin } \\
\text { Spain }\end{array}$ & $\begin{array}{l}\text { Sachiko Matsuhashi } \\
\text { Japan }\end{array}$ \\
\hline $\begin{array}{l}\text { Yefim Manevich } \\
\text { USA }\end{array}$ & $\begin{array}{l}\text { Adrian Mariño-Enriquez } \\
\text { USA }\end{array}$ & $\begin{array}{l}\text { Ken Matsui } \\
\text { USA }\end{array}$ \\
\hline $\begin{array}{l}\text { Sylvain Manfredi } \\
\text { France }\end{array}$ & $\begin{array}{l}\text { Talar Markossian } \\
\text { USA }\end{array}$ & $\begin{array}{l}\text { Kazumasa Matsumoto } \\
\text { Japan }\end{array}$ \\
\hline $\begin{array}{l}\text { Sendurai Mani } \\
\text { USA }\end{array}$ & $\begin{array}{l}\text { Athina Markou } \\
\text { Greece }\end{array}$ & $\begin{array}{l}\text { Shingo Matsumoto } \\
\text { Japan }\end{array}$ \\
\hline $\begin{array}{l}\text { David Mankoff } \\
\text { USA }\end{array}$ & $\begin{array}{l}\text { Janina Markowska } \\
\text { Poland }\end{array}$ & $\begin{array}{l}\text { Yukinori Matsuo } \\
\text { Japan }\end{array}$ \\
\hline $\begin{array}{l}\text { Melissa Mannion } \\
\text { USA }\end{array}$ & $\begin{array}{l}\text { David Marples } \\
\text { UK }\end{array}$ & $\begin{array}{l}\text { Hideyasu Matsuyama } \\
\text { Japan }\end{array}$ \\
\hline $\begin{array}{l}\text { Ulrich Mansmann } \\
\text { Germany }\end{array}$ & $\begin{array}{l}\text { Iván Márquez-Rodas } \\
\text { Spain }\end{array}$ & $\begin{array}{l}\text { Matthias Matter } \\
\text { Switzerland }\end{array}$ \\
\hline $\begin{array}{l}\text { Li Mao } \\
\text { USA }\end{array}$ & $\begin{array}{l}\text { Fatima Martel } \\
\text { Portugal }\end{array}$ & $\begin{array}{l}\text { Ines Mattos } \\
\text { Brazil }\end{array}$ \\
\hline $\begin{array}{l}\text { Xinliang Mao } \\
\text { China }\end{array}$ & $\begin{array}{l}\text { Janet Martin } \\
\text { Australia }\end{array}$ & $\begin{array}{l}\text { Estella Matutes } \\
\text { Spain }\end{array}$ \\
\hline $\begin{array}{l}\text { Christophe Kamungu Mapendano } \\
\text { Denmark }\end{array}$ & $\begin{array}{l}\text { Robert Martin } \\
\text { USA }\end{array}$ & $\begin{array}{l}\text { Loredana Mauro } \\
\text { Italy }\end{array}$ \\
\hline $\begin{array}{l}\text { Jodi Maranchie } \\
\text { USA }\end{array}$ & $\begin{array}{l}\text { Javier Martin-Broto } \\
\text { Spain }\end{array}$ & $\begin{array}{l}\text { Felicity E B May } \\
\text { UK }\end{array}$ \\
\hline $\begin{array}{l}\text { Paola Marcato } \\
\text { Canada }\end{array}$ & $\begin{array}{l}\text { Juan Martinez } \\
\text { Spain }\end{array}$ & $\begin{array}{l}\text { Philippa May } \\
\text { UK }\end{array}$ \\
\hline $\begin{array}{l}\text { Federica Marchesi } \\
\text { Italy }\end{array}$ & $\begin{array}{l}\text { Claudia Martini } \\
\text { Italy }\end{array}$ & $\begin{array}{l}\text { Joel Mayerson } \\
\text { USA }\end{array}$ \\
\hline $\begin{array}{l}\text { Julian Marchesi } \\
\text { UK }\end{array}$ & $\begin{array}{l}\text { Kouji Maruyama } \\
\text { Japan }\end{array}$ & $\begin{array}{l}\text { Eleni Mayson } \\
\text { Australia }\end{array}$ \\
\hline $\begin{array}{l}\text { Sergio Marchini } \\
\text { Italy }\end{array}$ & $\begin{array}{l}\text { Atsushi Masamune } \\
\text { Japan }\end{array}$ & $\begin{array}{l}\text { Pawel Mazur } \\
\text { USA }\end{array}$ \\
\hline
\end{tabular}


Guillermo Mazzolini

Argentina

Sam Mbulaiteye

USA

Florencia McAllister

USA

Shelli McAlpine

Australia

Pamela McCall

UK

Georgia McCann

USA

Damian McCartan

Ireland

Anne Marie McCarthy

USA

James A McCaul

UK

Sara McComb

USA

Carmel McConville

UK

Paula McDonald

UK

Paul McDonald

Canada

Braden McFarland

USA

Ronald McGarry

USA

Mark McKeage

New Zealand

Margaret McLaughlin-Drubin

USA

Philip McLoone

UK

Megan McNally

USA

Mairead McNamara

Canada

Stephen McPherson

Australia

Amy McQueen

USA
Justin McWilliams

USA

Alan Meeker

USA

Michal Mego

USA

Gabor Mehes

Hungary

Rohit Mehra

USA

Shikhar Mehrotra

USA

Davide Melisi

Italy

Anders Mellemgaard

Denmark

Lighua Meng

China

Li Rong Meng

Macao

Maxwell Meng

USA

Wei Meng

China

Lourdes Mengual

Spain

Kavitha Menon

New Zealand

Rolf Mentlein

Germany

Andres Merits

Estonia

Marco Carlo Merlano

Italy

Douglas Scott Merrell

USA

Megan Merrill

USA

Wilma Mesker

Netherlands

Giuseppe Alessio Messano Italy

Giulio Metro

Italy
Delia Mezzanzanica

Italy

Francesca Micci

Norway

George Michalopoulos

USA

Gaetane Michaud

USA

Pellegrino Michele

Italy

Mark Middleton

UK

James Mier

USA

Antimo Migliaccio

Italy

Lucia Migliore

Italy

Atsushi Miki

Japan

Wolfgang Mikulits

Austria

Branko Miladinovic

USA

Michele Milella

Italy

David Miles

UK

Donald Miller

USA

William Miller

UK

Frederick Millham

USA

Abul Milton

Australia

Seigo Minami

Japan

Marek Minarik

USA

Christoph Minichsdorfer

Austria

Pamela Minicozzi

Italy 


\author{
Cindy Miranti \\ USA
}

Sameer Mirza

USA

Shunichi Misawa

Japan

Anjali Mishra

India

Dhruva Kumar Mishra

USA

Anand Kumar Mishra

India

Shiraz Mishra

USA

Akira Mitsuhashi

Japan

Makito Miyake

Japan

Hiroshi Miyamoto

USA

Akihiko Miyanaga

Japan

Yoshihiro Miyasaka

Japan

Minoru Miyashita

Japan

Yoshihiko Miyata

Japan

Keiko Miyoshi

Japan

Xianming Mo

China

Helmout Modjtahedi

UK

Markus Moehler

Germany

Cathy Moelans

Netherlands

Siver Andreas Moestue

Norway

Maddalena Mognato

Italy

Mohammad Mohammadianpanah Iran
Altaf Mohammed

USA

Daniela Molena

USA

Alessio Molfino

Italy

Manuel Molina

USA

Sonia Molina-Pinelo

Spain

Francesca Molinari

Switzerland

Pål Møller

Norway

Chiara Mondello

Italy

Bradley Monk

USA

Ali Montazeri

Iran

Filippo Montemurro

Italy

Corey Montgomery

USA

Rodolfo Montironi

Italy

Dan Moore

USA

Roger Moorehead

Canada

Martin Mørck Mortensen

Denmark

James Morden

UK

Carlos Moreno

USA

Victor Moreno

Spain

Eiichi Morii

Japan

Toshikazu Moriwaki

Japan

Laura Moro

Italy
Denis Moro-Sibilot

France

David Morris

Australia

Joanna Moschandreas

Greece

Joachim Mössner

Germany

Aldo Mottino

Argentina

Marcella Mottolese

Italy

Giannis Mountzios

France

Mirna Mourtada-Maarabouni

UK

Mohamed Moustafa

Lebanon

Marek Mraz

Czech Republic

Ewa Mrozek

USA

David Mu

USA

Colin Muirhead

UK

Ken-Ichi Mukaisho

Japan

Arnab Mukherjea

USA

Malini Mukherjee

USA

Yoshiki Mukudai

Japan

Catherine Muller

France

Cecile Muller

Australia

Patricia Muller

UK

Sandra Muñoz-Galván

Spain

Alastair Munro

UK 


\author{
Jordi Muntané \\ Spain \\ Takashi Murakami \\ Japan \\ Raul Murillo \\ Colombia \\ Mandi Murph \\ USA \\ Jamie Murphy \\ UK \\ William Murphy \\ USA
}

Graeme Murray

UK

Douglas Murrey

USA

Charles Murtaugh

USA

Muhammed Murtaza

USA

Teemu Murtola

Finland

Lucia Anna Muscarella Jordan

Peter Muscarella

USA

Anna Maria Musti

Italy

Domenica Musumeci

Italy

Rajaram Nagarajan

USA

Pratik Nagaria

USA

Clara Nahmias

France

Shan Naidu

USA

Norifumi Naka

Japan

Mitsutoshi Nakada

Japan

Ikuhiko Nakase

Japan
Tetsuya Nakatsura

Japan

Tomio Nakayama

Japan

Harikrishna Nakshatri

USA

Joo-Hyun Nam

South Korea

Nisana Namwat

Thailand

Marius Nap

Netherlands

Gopeshwar Narayan

USA

Steven Narod

Canada

Tatiana Nasedkina

Russian Federation

Rachael Natrajan

UK

Shoji Natsugoe

Japan

Inna Naumov

Israel

Pierina Navarria

Italy

Alfons Navarro

Spain

Silvio Naviglio

Italy

Lakshmi Nayak

Uganda

Richard Neal

UK

Theo Nell South

Africa

Erik Nelson

USA

Camilla Nero

Italy

Jochen Neuhaus

Germany

Jens Neumann

Germany
Bart Neyns

Belgium

Jennifer Nicholas

UK

Anthony Nichols

Canada

Simone Niclou

Luxembourg

Alcina Nicol

Brazil

Carlos Nicolau

Spain

Francesco Nicolini

UK

Anders Lade Nielsen

Denmark

Finn Cilius Nielsen

Denmark

Torsten Nielsen

Canada

Giuseppe Nigri

Italy

Yuzuru Niibe

Japan

Hiroaki Niiro

Japan

Jonas Nilsson

Sweden

Mikael Nilsson

Sweden

Katharina Nimptsch

USA

Noriyuki Nishimura

USA

Tomohiro Nishina

Japan

Benjamin Nisman

Israel

Xiaohui Niu

China

Na Niu

USA

Sisse Helle Njor

Denmark 


\begin{tabular}{|c|c|c|}
\hline $\begin{array}{l}\text { Makoto Noda } \\
\text { Japan }\end{array}$ & $\begin{array}{l}\text { Akinyemi Ojesina } \\
\text { USA }\end{array}$ & $\begin{array}{l}\text { Hiroshi Onishi } \\
\text { Japan }\end{array}$ \\
\hline $\begin{array}{l}\text { Véronique Noé } \\
\text { Spain }\end{array}$ & $\begin{array}{l}\text { Yusuke Oji } \\
\text { Japan }\end{array}$ & $\begin{array}{l}\text { Maurizio Onisto } \\
\text { Italy }\end{array}$ \\
\hline $\begin{array}{l}\text { Lucien Noens } \\
\text { Belgium }\end{array}$ & $\begin{array}{l}\text { Hidenori Ojima } \\
\text { Japan }\end{array}$ & $\begin{array}{l}\text { Betul Oran } \\
\text { USA }\end{array}$ \\
\hline $\begin{array}{l}\text { Maria Claudia Nogueira Zerbini } \\
\text { Brazil }\end{array}$ & $\begin{array}{l}\text { Hirohisa Okabe } \\
\text { Japan }\end{array}$ & $\begin{array}{l}\text { Pedro Orihuela } \\
\text { Chile }\end{array}$ \\
\hline $\begin{array}{l}\text { Dong-Young Noh } \\
\text { South Korea }\end{array}$ & $\begin{array}{l}\text { Kinya Okamoto } \\
\text { Japan }\end{array}$ & $\begin{array}{l}\text { Josep Oriola } \\
\text { Spain }\end{array}$ \\
\hline $\begin{array}{l}\text { Sung Hoon Noh } \\
\text { South Korea }\end{array}$ & $\begin{array}{l}\text { Alicia Okines } \\
\text { UK }\end{array}$ & $\begin{array}{l}\text { Rosaria Orlandi } \\
\text { Italy }\end{array}$ \\
\hline $\begin{array}{l}\text { Nijiro Nohata } \\
\text { USA }\end{array}$ & $\begin{array}{l}\text { Natsuko Okita } \\
\text { Japan }\end{array}$ & $\begin{array}{l}\text { Daniel Orringer } \\
\text { USA }\end{array}$ \\
\hline $\begin{array}{l}\text { Takashi Nomiyama } \\
\text { Japan }\end{array}$ & $\begin{array}{l}\text { Yusuke Okuma } \\
\text { Japan }\end{array}$ & $\begin{array}{l}\text { Richard Orton } \\
\text { UK }\end{array}$ \\
\hline $\begin{array}{l}\text { Helena Nordenstedt } \\
\text { Sweden }\end{array}$ & $\begin{array}{l}\text { Lajos Olasz } \\
\text { Hungary }\end{array}$ & $\begin{array}{l}\text { Mitsuhiko Osaki } \\
\text { Japan }\end{array}$ \\
\hline $\begin{array}{l}\text { Nicola Normanno } \\
\text { Italy }\end{array}$ & $\begin{array}{l}\text { Raymond Oliphant } \\
\text { UK }\end{array}$ & $\begin{array}{l}\text { Daniel O'Shannessy } \\
\text { USA }\end{array}$ \\
\hline $\begin{array}{l}\text { Daniel Nowak } \\
\text { Germany }\end{array}$ & $\begin{array}{l}\text { Carla Oliveira } \\
\text { Portugal }\end{array}$ & $\begin{array}{l}\text { Sergej P. Osinsky } \\
\text { Ukraine }\end{array}$ \\
\hline $\begin{array}{l}\text { Ula Nur } \\
\text { UK }\end{array}$ & $\begin{array}{l}\text { Cristina Oliveira } \\
\text { Brazil }\end{array}$ & $\begin{array}{l}\text { Marie Stampe Ostenfeld } \\
\text { Denmark }\end{array}$ \\
\hline $\begin{array}{l}\text { Mari Nygård } \\
\text { Norway }\end{array}$ & $\begin{array}{l}\text { Leticia Oliveira-Ferrer } \\
\text { Germany }\end{array}$ & $\begin{array}{l}\text { Silvia Ottaviani } \\
\text { UK }\end{array}$ \\
\hline $\begin{array}{l}\text { Irena Oblak } \\
\text { Slovenia }\end{array}$ & $\begin{array}{l}\text { Lisa Oliver } \\
\text { France }\end{array}$ & $\begin{array}{l}\text { Leo Otterbein } \\
\text { USA }\end{array}$ \\
\hline $\begin{array}{l}\text { Dan O'Connell } \\
\text { Canada }\end{array}$ & $\begin{array}{l}\text { Micheau Olivier } \\
\text { France }\end{array}$ & $\begin{array}{l}\text { Penelope Ottewell } \\
\text { UK }\end{array}$ \\
\hline $\begin{array}{l}\text { James O'Connor } \\
\text { UK }\end{array}$ & $\begin{array}{l}\text { Jordan Olson } \\
\text { USA }\end{array}$ & $\begin{array}{l}\text { Laura Ottini } \\
\text { Italy }\end{array}$ \\
\hline $\begin{array}{l}\text { Ina Oehme } \\
\text { Germany }\end{array}$ & $\begin{array}{l}\text { Robert Olson } \\
\text { Canada }\end{array}$ & $\begin{array}{l}\text { Mototsugu Oya } \\
\text { Japan }\end{array}$ \\
\hline $\begin{array}{l}\text { Anthonia Ogbera } \\
\text { Nigeria }\end{array}$ & $\begin{array}{l}\text { Ian Olver } \\
\text { Australia }\end{array}$ & $\begin{array}{l}\text { Agostino Paccagnella } \\
\text { Italy }\end{array}$ \\
\hline $\begin{array}{l}\text { Shuji Ogino } \\
\text { USA }\end{array}$ & $\begin{array}{l}\text { David Omalley } \\
\text { USA }\end{array}$ & $\begin{array}{l}\text { Leisl Packer } \\
\text { Australia }\end{array}$ \\
\hline $\begin{array}{l}\text { Daniel Oh } \\
\text { USA }\end{array}$ & $\begin{array}{l}\text { Suzanne O'Neill } \\
\text { USA }\end{array}$ & $\begin{array}{l}\text { Roman Paduch } \\
\text { Poland }\end{array}$ \\
\hline $\begin{array}{l}\text { Robert Ohgami } \\
\text { USA }\end{array}$ & $\begin{array}{l}\text { Jason Ong } \\
\text { Australia }\end{array}$ & $\begin{array}{l}\text { Gabriella Pagnan } \\
\text { Italy }\end{array}$ \\
\hline $\begin{array}{l}\text { Kouki Ohtsuka } \\
\text { Japan }\end{array}$ & $\begin{array}{l}\text { Evan Ong } \\
\text { USA }\end{array}$ & $\begin{array}{l}\text { Katia Pagnano } \\
\text { Brazil }\end{array}$ \\
\hline
\end{tabular}


Young-Ki Paik

South Korea

Tuya Pal

USA

Pierlorenzo Pallante Italy

Athanasios Pallis

Greece

Trenis Palmer

USA

Marta Palmieri

Italy

Moritz Palmowski

Germany

Grazia Palomba

Italy

Jianji Pan

China

Jingxuan Pan

China

Sai Yi Pan

Canada

Katherine Panageas

USA

Vijay Pandey

Singapore

Gopi Krishna Panicker

India

Carolina Panis

Brazil

Kostas Pantopoulos

Canada

Alexandros Papalambros

Greece

George Papaxoinis

Greece

Laura Papi

Emmanouil Pappou

USA

Angelo Paradiso

Italy

Bhupesh Parashar

USA
Christos Paraskeva

UK

Olivier Pardo

UK

Rahul Parikh

USA

Carol Parise

USA

Changwon Park

USA

Hee Chul Park

South Korea

Jinha Park

USA

Seho Park

South Korea

Kyu-Sang Park

South Korea

Sook Ryun Park

South Korea

Maurizio Parola

Italy

Paola Parrella

Italy

Luca Parrillo

Italy

Toshima Parris

Sweden

Barbara Parsons

USA

Valeria Pascale

Italy

Steve Pascolo

Germany

Eddy Pasquier

Australia

Marilo Pastor

Spain

Sandra Pastorino

USA

Roberta Pastorino Italy

Asmita Patel

New Zealand
Jitesh Patel

USA

Maitraya Patel

USA

Prabhudas Patel

India

Nitin Patil

Germany

Deepa Patil

USA

Vijay Patil

India

Ruth Patterson

USA

Nicholas Pavlidis

Greece

Laura Pazzaglia

Italy

Jing Pei

China

Helene Pelicano

USA

Jerry Pelletier

Canada

Juha Peltonen

Finland

Du Peng

China

Fangyu Peng

USA

Xiaohua Peng

USA

Jun Peng

China

Marzia Pennati

Italy

Núbia Braga Pereira

Brazil

Francesco Perrone

Italy

Federica Perrone

Italy

Luca Persano

Italy 
Manuel Perucho

USA

Janette Perz

Australia

Patrick Petignat

Switzerland

Stefano Petti

Italy

Vincenzo Pezzi

Italy

Claudia Pföhler

Germany

Prakash Babu Phanithi

India

Lynette Phillips

USA

Gary Piazza

USA

Giulia Piazzi

Italy

Maria Carmela Piccirillo

Italy

Alain Piche

Canada

Martin Pichler

Austria

Phillip Pierorazio

USA

Pages Pierre-Benoit

France

Maria Catherine Pietanza

USA

Thierry Pieters

Belgium

Filippo Pietrantonio

Italy

Melissa Pilewskie

USA

Radhakrishna Pillai

India

Dietmar Pils

Austria

Pascal Pineau

France
Benjamin Pineda

Mexico

Céline Pinheiro

Brazil

Maria Simona Pino

Italy

Ruben Pio

Spain

Grzegorz Piotrowski

Poland

Martin Pirkl

Germany

Jose Piruat

Spain

Salvatore Piscuoglio

USA

Vincenzo Pitini

Italy

Sara Pizzamiglio

Italy

Laura Pizzuti

Italy

Andrzej Plawski

Poland

Ruben Plentz

Germany

Miklos Pless

Switzerland

Ferdinand Ploner

Austria

Janice Pluth

USA

Martin Pölcher

Germany

Hans-Christian Pommergaard

Denmark

Mathurose Ponglikitmongkol

Thailand

Brenten Popiel

USA

Helena Pópulo

Portugal

Luca Porcu

Italy
Paola Postacchini

Italy

David Potter

USA

George Poultsides

USA

Philippe Pourquier

France

Ned Powell

UK

Emily Power

UK

Samantha Pozzi

Italy

C Pramesh

India

Sandip Prasad

USA

Aleix Prat

Spain

Sarah Preisler

Denmark

Hans Prenen

Belgium

Ana Preto

Portugal

Allan Price

UK

Shawn Price

USA

Timothy Price

Australia

Ivanka Prichard

Australia

Alessandro Prinetti

Italy

Andrea Prodosmo

Italy

Sandi Pruitt

USA

Giancarlo Pruneri

Italy

Christopher Przybycin

USA 


\begin{tabular}{|c|c|c|}
\hline Dimitri Psimaras & Francesca Raimondo & Anne Reiman \\
\hline France & Italy & UK \\
\hline Ashok Pullikuth & Michal Rajski & Hong Ren \\
\hline USA & Switzerland & China \\
\hline Marco Pupo & Janusz Rak & Noa Rensing \\
\hline Italy & Canada & USA \\
\hline Michele Purrello & Stephen Ramsey & Diana Resendez \\
\hline Italy & USA & Mexico \\
\hline Brigitte Pützer & Guglielmina Ranzani & Fabien Reyal \\
\hline Germany & Italy & France \\
\hline Wei-Xiang Qi & Ganesh Rao & Carolina Reyes \\
\hline China & USA & USA \\
\hline Chengyong Qin & Rajini Rao & Jordan Reynolds \\
\hline China & USA & USA \\
\hline Xue Qin & Kati Rasanen & Domenico Ribatti \\
\hline China & Finland & Italy \\
\hline Jingxin Qiu & Dominique Rash & Carmela Ricciardelli \\
\hline USA & USA & Australia \\
\hline Xiujuan Qu & Timothy Ratliff & Jeremy Rich \\
\hline China & USA & USA \\
\hline Marcelo Queiroz & Elena Ratner & Holger Richly \\
\hline Switzerland & USA & Germany \\
\hline Richard Quek & Edward Ratovitski & Christopher Ricketts \\
\hline Singapore & USA & USA \\
\hline Hilmar Quentmeier & Tilman Rau & Johannes Rieger \\
\hline Germany & Switzerland & Germany \\
\hline Victor Quesada & Paul Rava & Piotr Rieske \\
\hline Spain & USA & Poland \\
\hline Peter Quesenberry & Pritha Ray & Jonathan Riess \\
\hline USA & India & USA \\
\hline Andrew Quest & Karine Raymond & Oliver Riesterer \\
\hline Chile & France & Switzerland \\
\hline Patrick Quint & Matejka Rebolj & Michel Rigaud \\
\hline USA & Denmark & France \\
\hline Jürgen Radons & Diana Redwood & Guido Rindi \\
\hline Germany & USA & Italy \\
\hline Milan Radovich & Katherine Reeves & Michael Risk \\
\hline USA & USA & USA \\
\hline Colin Rae & David Regan & Susan Rittling \\
\hline UK & Australia & USA \\
\hline Sajjad Rafiq & Enrique Regidor & Juan Carklos Roa \\
\hline UK & Spain & Chile \\
\hline Nuh Rahbari & Alistair Reid & Karin Roberg \\
\hline Germany & UK & Sweden \\
\hline
\end{tabular}


Jacques Robert

France

Ryan Roberts

USA

Keith Robertson

USA

Kim Robien

USA

Cliff Robinson

USA

Trude Eid Robsahm

Norway

Mark Robson

USA

Marco Rocchi

Italy

Sonia Rocha

UK

Franz Rödel

Germany

Rb Roden

USA

Wendy Rodenburg

Netherlands

Mauricio Rodriguez Dorantes

Mexico

Socorro Maria Rodriguez Pinilla

Spain

Claire Rodriguez-Lafrasse

France

Achim Rody

Germany

Falk Roeder

Germany

Bernard Roelen

Netherlands

Sebastian Rogenhofer

Germany

Simon Rogers

UK

Stephen Rohan

USA

Christian Rolfo

Belgium
Maurizio Romano

Italy

Margarita Romeo

Spain

Chiara Romualdi

Italy

Minhua Rong

China

Dana Roque

USA

Antonia Roseweir

UK

Antonella Rosi

Italy

Stephanie Rosse

USA

Charles Rosser

USA

Sabrina Rossi

Jordan

Matteo Rota

Italy

Johann Rotheneder

Austria

Etienne Rouleau

France

Jason Roy

USA

Shreyas Roy

USA

Susanta Roychoudhury

India

Giovina Ruberti

Italy

Luca Rubino

Italy

Randall Ruch

USA

Volker Rudat Saudi

Arabia

Christian Rudlowski

Germany

Emil Rudolf

Czech Republic
Anja Rudolph

Germany

Montserrat Rue

Spain

Claudia Ruebe

Germany

Corina S. Rueegg

Switzerland

Thomas Ruhstaller

Switzerland

Alvaro Ruibal

Spain

Roslin Russell

UK

Martin Ruthardt

Germany

Jim Rutka

Canada

Munindra Ruwali

India

Lisa Rydén

Sweden

Sepideh Saadatmand

Netherlands

Kauko Samuel Saarilahti

Finland

Renaud Sabatier

France

Vera Saddi

Brazil

Andrea Sadlonova

USA

Toshiaki Saeki

Japan

Carmen Saez

Spain

Motoyasu Sagawa

Japan

Manujendra Saha

Canada

Solmaz Sahebjam

USA

Abdul Saied

USA 


\begin{tabular}{|c|c|c|}
\hline $\begin{array}{l}\text { Neeru Saini } \\
\text { India }\end{array}$ & $\begin{array}{l}\text { Matteo Santoni } \\
\text { Italy }\end{array}$ & $\begin{array}{l}\text { Beat Schäfer } \\
\text { Switzerland }\end{array}$ \\
\hline $\begin{array}{l}\text { Yoshifumi Saisho } \\
\text { Japan }\end{array}$ & $\begin{array}{l}\text { Eric Santoni-Rugiu } \\
\text { Denmark }\end{array}$ & $\begin{array}{l}\text { Florence Schaffner } \\
\text { France }\end{array}$ \\
\hline $\begin{array}{l}\text { Hiroshi Saito } \\
\text { Japan }\end{array}$ & $\begin{array}{l}\text { Sankar Sanyal } \\
\text { India }\end{array}$ & $\begin{array}{l}\text { Helmut Schaider } \\
\text { Australia }\end{array}$ \\
\hline $\begin{array}{l}\text { Kazutaka Saito } \\
\text { Japan }\end{array}$ & $\begin{array}{l}\text { Gabriele Saretzki } \\
\text { UK }\end{array}$ & $\begin{array}{l}\text { Catherine Schairer } \\
\text { USA }\end{array}$ \\
\hline $\begin{array}{l}\text { Yuki Saito } \\
\text { Japan }\end{array}$ & $\begin{array}{l}\text { Luis Sarian } \\
\text { Brazil }\end{array}$ & $\begin{array}{l}\text { Marilyn Schapira } \\
\text { USA }\end{array}$ \\
\hline $\begin{array}{l}\text { Masao Saitoh } \\
\text { Japan }\end{array}$ & $\begin{array}{l}\text { Sulen Sarioglu } \\
\text { Turkey }\end{array}$ & $\begin{array}{l}\text { Tom Scharschmidt } \\
\text { USA }\end{array}$ \\
\hline $\begin{array}{l}\text { Eiji Sakai } \\
\text { Japan }\end{array}$ & $\begin{array}{l}\text { Balazs Sarkadi } \\
\text { Hungary }\end{array}$ & $\begin{array}{l}\text { Laura Scherer } \\
\text { USA }\end{array}$ \\
\hline $\begin{array}{l}\text { Yasunaru Sakuma } \\
\text { Japan }\end{array}$ & $\begin{array}{l}\text { Ana Bela Sarmento-Ribeiro } \\
\text { Portugal }\end{array}$ & $\begin{array}{l}\text { Roxana Schillaci } \\
\text { Argentina }\end{array}$ \\
\hline $\begin{array}{l}\text { Ritu Salani } \\
\text { USA }\end{array}$ & $\begin{array}{l}\text { Pablo Sarobe } \\
\text { Spain }\end{array}$ & $\begin{array}{l}\text { Christoph Schlapbach } \\
\text { Switzerland }\end{array}$ \\
\hline $\begin{array}{l}\text { Dolores Salas } \\
\text { Spain }\end{array}$ & $\begin{array}{l}\text { Aaron Sarver } \\
\text { USA }\end{array}$ & $\begin{array}{l}\text { Paul G Schlegel } \\
\text { Germany }\end{array}$ \\
\hline $\begin{array}{l}\text { Mauricio Salcedo } \\
\text { Mexico }\end{array}$ & $\begin{array}{l}\text { Shizuka Sasazuki } \\
\text { Japan }\end{array}$ & $\begin{array}{l}\text { Martina Schmidt } \\
\text { Netherlands }\end{array}$ \\
\hline $\begin{array}{l}\text { Saima Saleem } \\
\text { Pakistan }\end{array}$ & $\begin{array}{l}\text { Takami Sato } \\
\text { USA }\end{array}$ & $\begin{array}{l}\text { Fernando Schmitt } \\
\text { Portugal }\end{array}$ \\
\hline $\begin{array}{l}\text { Nikolaos Salemis } \\
\text { Greece }\end{array}$ & $\begin{array}{l}\text { Taroh Satoh } \\
\text { Japan }\end{array}$ & $\begin{array}{l}\text { Wolfgang Schmitt } \\
\text { Germany }\end{array}$ \\
\hline $\begin{array}{l}\text { David Saltman } \\
\text { Canada }\end{array}$ & $\begin{array}{l}\text { Vishnupriya Satti } \\
\text { India }\end{array}$ & $\begin{array}{l}\text { Marc Schmitz } \\
\text { Germany }\end{array}$ \\
\hline $\begin{array}{l}\text { Vanda Salutari } \\
\text { Italy }\end{array}$ & $\begin{array}{l}\text { Torill Sauer } \\
\text { Norway }\end{array}$ & $\begin{array}{l}\text { Paul Magnus Schneider } \\
\text { Switzerland }\end{array}$ \\
\hline $\begin{array}{l}\text { Ignacio San Francisco } \\
\text { Chile }\end{array}$ & $\begin{array}{l}\text { Niramol Savaraj } \\
\text { USA }\end{array}$ & $\begin{array}{l}\text { Birgit Schoeberl } \\
\text { USA }\end{array}$ \\
\hline $\begin{array}{l}\text { Margarita Sanchez-Beato } \\
\text { Spain }\end{array}$ & $\begin{array}{l}\text { Norie Sawada } \\
\text { Japan }\end{array}$ & $\begin{array}{l}\text { Markus Scholz } \\
\text { Germany }\end{array}$ \\
\hline $\begin{array}{l}\text { Rupninder Sandhu } \\
\text { USA }\end{array}$ & $\begin{array}{l}\text { Okay Saydam } \\
\text { Austria }\end{array}$ & $\begin{array}{l}\text { Mario Schootman } \\
\text { USA }\end{array}$ \\
\hline $\begin{array}{l}\text { Juan Sandoval } \\
\text { Spain }\end{array}$ & $\begin{array}{l}\text { Bruna Scaggiante } \\
\text { Italy }\end{array}$ & $\begin{array}{l}\text { Andres Jan Schrader } \\
\text { Germany }\end{array}$ \\
\hline $\begin{array}{l}\text { Sumathi Sankaran-Walters } \\
\text { USA }\end{array}$ & $\begin{array}{l}\text { Aldo Scarpa } \\
\text { Italy }\end{array}$ & $\begin{array}{l}\text { Michael Georg Schrauder } \\
\text { Germany }\end{array}$ \\
\hline $\begin{array}{l}\text { Sampath Santhosh } \\
\text { India }\end{array}$ & $\begin{array}{l}\text { Karin Scarpinato } \\
\text { USA }\end{array}$ & $\begin{array}{l}\text { Michael Schuler } \\
\text { Germany }\end{array}$ \\
\hline $\begin{array}{l}\text { Marta Santisteban } \\
\text { Spain }\end{array}$ & $\begin{array}{l}\text { Michael Schaapveld } \\
\text { Netherlands }\end{array}$ & $\begin{array}{l}\text { Linda Schuler } \\
\text { USA }\end{array}$ \\
\hline
\end{tabular}




\begin{tabular}{|c|c|c|}
\hline Wolfgang Schulz & Raquel Seruca & Lizong Shen \\
\hline Germany & Portugal & China \\
\hline Udo Schumacher & Gautam Sethi & Ke Sheng \\
\hline Germany & USA & USA \\
\hline Eric Schuur & Bhuvana Setty & Elizabeth Shephard \\
\hline USA & USA & UK \\
\hline Gerrit Schuurhuis & Patricia Severino & Trevor Shepherd \\
\hline Netherlands & Brazil & Canada \\
\hline Thomas Schwaab & Alex Sgambato & Kirti Shetty \\
\hline USA & Italy & USA \\
\hline Lee Schwartzberg & Mehdi Shakibaei & Runhua Shi \\
\hline USA & Germany & USA \\
\hline Heidi Schwarzenbach & Ali Shamseddine & Ruihua Shi \\
\hline Germany & Lebanon & China \\
\hline Lukas Schwentner & Xiying Shang & Ming Shi \\
\hline Germany & USA & China \\
\hline Kathryn Schwertfeger & Jieya Shao & Hisayuki Shigematsu \\
\hline USA & USA & Japan \\
\hline Andreas Scorilas & Anna Shapiro & Jin-Yuan Shih \\
\hline Greece & USA & Taiwan \\
\hline Suzanne Scott & Shashwat Sharad & Naoto Shikama \\
\hline UK & USA & Japan \\
\hline Anna Ivana Scovassi & Akash Sharma & Takeo Shimasaki \\
\hline Italy & USA & Japan \\
\hline Beatrice Seddon & Dipali Sharma & Shoji Shimoyama \\
\hline UK & USA & Japan \\
\hline Carmen Segrelles Huelga & Linda Sharp & Myung-Geun Shin \\
\hline Spain & Ireland & South Korea \\
\hline Masahiro Seike & Leslie Shaw & Hai-Rim Shin \\
\hline Japan & USA & Philippines \\
\hline Takahiro Seki & Richard Shaw & Yoshiyuki Shioyama \\
\hline Sweden & UK & Japan \\
\hline Michaela Semeraro & Paul Shaw & Hidenori Shiraha \\
\hline France & UK & Japan \\
\hline Bo Kyoung Seo & Kieran Sheahan & Kohei Shitara \\
\hline South Korea & Italy & Japan \\
\hline Yeon Sun Seong & Jason Sheehan & Yow-Ling Shiue \\
\hline South Korea & USA & Taiwan \\
\hline Massimo Serra & Bairong Shen & Farbod Shojaei \\
\hline Italy & China & USA \\
\hline$M^{\text {a }}$ Jose Serrano & Rulong Shen & Hirokazu Shoji \\
\hline Spain & USA & Japan \\
\hline Rosario Serrano & Yaxing Shen & Lynette Sholl \\
\hline Spain & China & USA \\
\hline
\end{tabular}




\begin{tabular}{|c|c|c|}
\hline $\begin{array}{l}\text { Noam Shomron } \\
\text { Israel }\end{array}$ & $\begin{array}{l}\text { Sheila Singh } \\
\text { Canada }\end{array}$ & $\begin{array}{l}\text { Artem Sokolov } \\
\text { USA }\end{array}$ \\
\hline $\begin{array}{l}\text { Susan Short } \\
\text { UK }\end{array}$ & $\begin{array}{l}\text { Aatur Singhi } \\
\text { USA }\end{array}$ & $\begin{array}{l}\text { Maria Soldatkina } \\
\text { Ukraine }\end{array}$ \\
\hline $\begin{array}{l}\text { Mohamed Shoukri } \\
\text { Saudi Arabia }\end{array}$ & $\begin{array}{l}\text { Prem Sinha } \\
\text { USA }\end{array}$ & $\begin{array}{l}\text { Xavier Sole } \\
\text { Spain }\end{array}$ \\
\hline $\begin{array}{l}\text { Samia Shouman } \\
\text { Egypt }\end{array}$ & $\begin{array}{l}\text { Birandra Sinha } \\
\text { USA }\end{array}$ & $\begin{array}{l}\text { Ponnandai Somasundar } \\
\text { USA }\end{array}$ \\
\hline $\begin{array}{l}\text { Viji Shridhar } \\
\text { USA }\end{array}$ & $\begin{array}{l}\text { Ronit Sionov } \\
\text { Israel }\end{array}$ & $\begin{array}{l}\text { Alvaro Somoza } \\
\text { Spain }\end{array}$ \\
\hline $\begin{array}{l}\text { Stuti Shroff } \\
\text { USA }\end{array}$ & $\begin{array}{l}\text { Alphonse Sirica } \\
\text { USA }\end{array}$ & $\begin{array}{l}\text { Yong Song } \\
\text { China }\end{array}$ \\
\hline $\begin{array}{l}\text { Ting Si } \\
\text { China }\end{array}$ & $\begin{array}{l}\text { Diego Sisci } \\
\text { Italy }\end{array}$ & $\begin{array}{l}\text { Yong Sang Song } \\
\text { South Korea }\end{array}$ \\
\hline $\begin{array}{l}\text { Kenneth Siddle } \\
\text { UK }\end{array}$ & $\begin{array}{l}\text { Shankar Siva } \\
\text { Australia }\end{array}$ & $\begin{array}{l}\text { Guru Sonpavde } \\
\text { USA }\end{array}$ \\
\hline $\begin{array}{l}\text { Lucas Sideris } \\
\text { Canada }\end{array}$ & $\begin{array}{l}\text { Tobias Sjöblom } \\
\text { Sweden }\end{array}$ & $\begin{array}{l}\text { Richie Soong } \\
\text { Singapore }\end{array}$ \\
\hline $\begin{array}{l}\text { Anna Sidorchuk } \\
\text { Sweden }\end{array}$ & $\begin{array}{l}\text { James Skipworth } \\
\text { UK }\end{array}$ & $\begin{array}{l}\text { Prasanna Sooriakumaran } \\
\text { UK }\end{array}$ \\
\hline $\begin{array}{l}\text { Gene Siegal } \\
\text { USA }\end{array}$ & $\begin{array}{l}\text { Frank Slack } \\
\text { USA }\end{array}$ & $\begin{array}{l}\text { Halfdan Sorbye } \\
\text { Norway }\end{array}$ \\
\hline $\begin{array}{l}\text { Robert Siegel } \\
\text { Germany }\end{array}$ & $\begin{array}{l}\text { Andrzej Slominski } \\
\text { USA }\end{array}$ & $\begin{array}{l}\text { Karina Dalsgaard Sorensen } \\
\text { Denmark }\end{array}$ \\
\hline $\begin{array}{l}\text { Cornelis Sier } \\
\text { Netherlands }\end{array}$ & $\begin{array}{l}\text { Peter Sminia } \\
\text { Netherlands }\end{array}$ & $\begin{array}{l}\text { Irma Soria-Mercado } \\
\text { Mexico }\end{array}$ \\
\hline $\begin{array}{l}\text { Luca Sigalotti } \\
\text { Italy }\end{array}$ & $\begin{array}{l}\text { Alexandra Smith } \\
\text { UK }\end{array}$ & $\begin{array}{l}\text { José Luis Soto } \\
\text { Spain }\end{array}$ \\
\hline $\begin{array}{l}\text { Ana Elizabete Silva } \\
\text { Brazil }\end{array}$ & $\begin{array}{l}\text { Bryan Smith } \\
\text { USA }\end{array}$ & $\begin{array}{l}\text { John Souglakos } \\
\text { Greece }\end{array}$ \\
\hline $\begin{array}{l}\text { Paulo Silveira } \\
\text { Brazil }\end{array}$ & $\begin{array}{l}\text { Gary Smith } \\
\text { USA }\end{array}$ & $\begin{array}{l}\text { Harmik Soukiasian } \\
\text { USA }\end{array}$ \\
\hline $\begin{array}{l}\text { Marko Simunovic } \\
\text { Canada }\end{array}$ & $\begin{array}{l}\text { Steven Smith } \\
\text { USA }\end{array}$ & $\begin{array}{l}\text { Paul Span } \\
\text { Netherlands }\end{array}$ \\
\hline $\begin{array}{l}\text { Alessandro Sindoni } \\
\text { Italy }\end{array}$ & $\begin{array}{l}\text { Peter Smyth } \\
\text { Ireland }\end{array}$ & $\begin{array}{l}\text { Valerie Speirs } \\
\text { UK }\end{array}$ \\
\hline $\begin{array}{l}\text { Amanpal Singh } \\
\text { USA }\end{array}$ & $\begin{array}{l}\text { Asley Snider } \\
\text { USA }\end{array}$ & $\begin{array}{l}\text { Claudio Spick } \\
\text { Austria }\end{array}$ \\
\hline $\begin{array}{l}\text { Pankaj Singh } \\
\text { USA }\end{array}$ & $\begin{array}{l}\text { Jonathan Soboloff } \\
\text { USA }\end{array}$ & $\begin{array}{l}\text { Gian Paolo Spinelli } \\
\text { Italy }\end{array}$ \\
\hline $\begin{array}{l}\text { Praby Singh } \\
\text { USA }\end{array}$ & $\begin{array}{l}\text { Silvia Soddu } \\
\text { Italy }\end{array}$ & $\begin{array}{l}\text { Gilbert Spizzo } \\
\text { Italy }\end{array}$ \\
\hline $\begin{array}{l}\text { Rakesh Singh } \\
\text { USA }\end{array}$ & $\begin{array}{l}\text { Elin Söderberg } \\
\text { Sweden }\end{array}$ & $\begin{array}{l}\text { Jeremy Squire } \\
\text { Brazil }\end{array}$ \\
\hline
\end{tabular}


Nahida Srabovic

Bosnia and Herzegovina

Sriganesh Srihari

India

Sanjay K. Srivastava

USA

Elvira Stacher

Austria

John Staffurth

UK

John Stagg

Canada

Venturina Stagni

Italy

Olle Stål

Sweden

Helge Stalsberg

Norway

Daniel Starczynowski

USA

Andreas Stark

Germany

Kristen Stashek

USA

Giorgio Stassi

Italy

Georgios Stathopoulos

Greece

Rafal Stec

Poland

Sandra Steffens

Germany

Guenther Steger

Austria

Eva Steliarova-Foucher

France

Ulf-Hakan Stenman

Finland

Nathan Stephens

UK

Richard Stevens

USA

Christopher Stevenson

Australia
Anton Stift

Austria

Charles Stiller

UK

Christopher Stipp

USA

Lucia Anna Stivala

Italy

Andrew Stoker

UK

Katja Storch

Germany

Douglas Strand

USA

Carina Strell

Sweden

Michael Stroehlein

Germany

Fay Strohschein

Canada

Darrin Stuart

USA

Dominik Sturm

Germany

Chun-Li Su

Taiwan

Rongjian Su

China

Changqing Su

China

Jin Su

China

Li-Jen Su

Taiwan

Subbaya Subramanian

USA

Takeshi Suda

Japan

Tamotsu Sugai

Japan

Tomoharu Sugie

Japan

Mitsushige Sugimoto

Japan
Haruhiko Sugimura

Japan

Kevin Sun

New Zealand

Shi-Yong Sun

USA

Jing Sun

China

Yihua Sun

China

Shaogang Sun

USA

Xinchen Sun

China

Yanan Sun

China

Wei Sun

China

Wenxiang Sun

China

Yu Sunakawa

USA

Debasish Sundi

USA

Joseph Sung

Hong Kong

Zhenhe Suo

Norway

Rajah Supramaniam

Australia

Alexey Surov

Germany

Larry Suva

USA

Akihiko Suzuki

Japan

Eiichiro Suzuki

Japan

Hidekazu Suzuki

Japan

Yutaka Suzuki

Japan

Rajaraman Swaminathan India 
Benjamin Swanson

USA

Colleen Sweeney

USA

Fred Sweep

Netherlands

Peter Szabo

USA

Marta Szajnik

Poland

Tibor Szarvas

Austria

Peter Szlosarek

UK

Krzysztof (Chris) Szyfter

Poland

Yoshiaki Tabuchi

Japan

Michael Tachezy

Germany

Yuji Tachimori

Japan

Keiichiro Tada

Japan

Elda Tagliabue

Italy

Pierosandro Tagliaferri

Italy

Alberto Tagliafico

Italy

Ayumu Taguchi

USA

Sk Tai

Taiwan

Daisuke Takahari

Japan

Ryuji Takahashi

Japan

Atsuo Takashima

Japan

Ken-Ichi Takayama

Japan

Tetsuo Takehara

Japan
Akinobu Taketomi

Japan

Kengo Takeuchi

Japan

Jun-Ichi Tamaru

Japan

Daniel Tan

Singapore

Eng Huat Tan

Singapore

Hiromi Tanaka

USA

Motoyoshi Tanaka

Japan

Cristiana Tanase

Romania

Kari Tanderup

USA

Naoko Tanese

USA

Dabei Tang

China

Hua Tang

China

Xinyu Tang

USA

Robert M. Tanguay

Canada

Philippe Taniere

UK

Pieter Tanis

Netherlands

Edward Tanner

USA

Tamara Tanos

Italy

Hideki Tanzawa

Japan

Lei Tao

China

Raquel Tarazona

Spain

Jussi Tarkkanen

Finland
Line Tarpgaard

Denmark

Natasha Tasevska

USA

Tyna Taskila

UK

Mine Islimye Taskin

USA

Pierfrancesco Tassone

Italy

Helge Taubert

Germany

Sun Kuie Tay

Singapore

Sally Taylor

UK

Georgi Tchernev

Bulgaria

Muy-Kheng M. Tea

Austria

Rajeshwar Rao Tekmal

USA

Teruhiko Terasawa

Japan

Evangelos Terpos

Greece

Luigi Terracciano

Switzerland

Mary Beth Terry

USA

Paul Terry

USA

Giovanni Tesoriere

Italy

Anna Tessari

USA

Krishnansu Tewari

USA

George Thalmann

Switzerland

Douglas Thamm

USA

Ekkasit Tharavichitkul

Thailand 
Kullathorn Thephamongkhol

Thailand

Brigitte Thériault

Canada

Anne Thiebaut

France

Susan Thomas

USA

Linda Thompson

USA

John Thoms

Canada

Mathilde Borg Houlberg Thomsen

Denmark

\section{Steven Thorpe \\ USA \\ Vesteinn Thorsson \\ USA}

Nirav Thosani

USA

Inger Thune

Norway

Peter Thuss-Patience

Germany

Bharat Thyagarajan

USA

Ivana Ticha

Czech Republic

Shweta Tikoo

Australia

David Timson

UK

Andrea Tinelli

Italy

Ingeborg Tinhofer

Germany

Shinsaku Togo

Japan

Chee-Keong Toh

Singapore

Yuji Toiyama

Japan

Arinobu Tojo

Japan
Rodrigo Toledo

USA

Nori Tolosa De Talamoni

Argentina

Federica Tomao

Italy

Marco Tomasetti

Italy

Yoshihiro Tominaga

Japan

Patricia Tonin

Canada

Jyoti Tope

India

Matthew Topham

USA

Erkan Topkan

Turkey

Paul Toren

Canada

Toshihiko Torigoe

Japan

Luigi Tornillo

Switzerland

Anna Torres

Poland

Alicia Torriglia

France

Suzy Torti

USA

Yann Touchefeu

France

Marina Touillaud

France

Amanda Townsend

Australia

Nham Tran

Australia

Olivier Tredan

France

Marjolijn Trietsch

Netherlands

Fabio Trippa

Italy
Tiziana Triulzi

Italy

Lucio Trodella

Italy

Volodymyr Tryndyak

USA

Kenneth Tsai

USA

Sen-Tien Tsai

Taiwan

Nadejda Tsankova

USA

George Sai-Wah Tsao

Hong Kong

Vassiliki Liana Tsikitis

USA

Konstantinos Tsilidis

Greece

George Tsirakis

Greece

Kelvin Tsoi

Hong Kong

Tung Yu Tsui

Germany

Takemasa Tsuji

USA

Ryouichi Tsunedomi

Japan

Nelson Hirokazu Tsuno

Japan

Jan Tuckermann

Germany

Musaffe Tuna

USA

Yusuf Tutar

USA

James Tysome

UK

George Tzanakakis

Greece

Flora Tzelepis

Australia

Keisuke Uehara

Japan 


Selma Ugurel
Germany
Eugen Uhlmann
Germany
Nancy Uhrhammer
France
Shigeki Umemura
Japan
Gerold Untergasser
Austria
Susanne Unverzagt
Germany

Ghanshyam Upadhyay

India

Cheerag Upadhyaya

USA

Jean-Luc Urbain

USA

Fyodor Urnov

USA

Ruchan Uslu

Turkey

Jaydutt Vadgama

USA

Ratna Vadlamudi

USA

Jayant Vaidya

UK

Antonis Valachis

Greece

Guido Valente

Italy

Daniel Vallbohmer

Germany

Sonia Vallet

Germany

Lampros Vamvakas

Greece

Charles Van Berlo

Netherlands

Elske Van Den Akker

Netherlands

Jasper G. Van Den Boorn

Germany
Donald L. Van Der Peet

Netherlands

W.A. Van Der Zwan

Netherlands

Carolien Van Deurzen

Netherlands

Carla Van Gils

Netherlands

Elke Van Hoof

Belgium

Leonie Van Hulsteijn

Netherlands

Marlous Van Laar

UK

Ryan Van Laar

USA

Marieke Van Leeuwen

Netherlands

Jos Van Pelt

Belgium

Joost Van Rosmalen

Netherlands

Michelle Van Ryn

USA

Peter Van Veldhuizen

USA

Marcel Van Vugt

Netherlands

Vincent Vander Poorten

Belgium

Greta Varchi

Italy

Marileila Varella-Garcia

USA

Andreas Varkaris

USA

John Varlotto

USA

Mohammad Vasei

Iran

Vasyl Vasko

USA

Theodoros Vassilakopoulos

Greece
Athanassios Vassilopoulos

USA

David Vaughn

USA

Peter Vaupel

Germany

Dominique Vaur

France

Mikhail Vaysberg

USA

Piret Veerus

Estonia

Vaneja Velenik

Slovenia

Sergia Velho

Portugal

Tiziana Venesio

Italy

Girish Venkataraman

USA

Marina Vercelli

Italy

Paolo Verderio

Italy

Douglas Vernimmen

UK

Maud Verstraete

Belgium

Andre Vettore

Brazil

Gustavo Viani

Brazil

Laura Vidal Boixader

Spain

Sergi Vidal Sicart

Spain

Andre F Vieira

Portugal

Giuseppe Viglietto

Italy

Riccardo Vigneri

Italy

Raffaella Villa

Italy 
Priam Villalonga

Spain

Piroska Virag

Romania

Pauline Vissers

Netherlands

Bella Vivat

UK

Francisco Vizoso

Spain

Giuseppe Vizzielli

Italy

Panagiotis Vlachostergios

USA

Virginie Vlaeminck-Guillem

France

Arndt Vogel

Germany

Michael Von Bergwelt-Baildon

Germany

André O. Von Bueren

Germany

Jw Voncken

Netherlands

Adri Voogd

Netherlands

Dirk Vordermark

Germany

Hilke Vorwerk

Germany

Mark Voskoboynik

UK

Gerassimos Voutsinas

Greece

Semir Vranic

Bosnia and Herzegovina

David Lukas Wachter

Germany

Jamie Wagner

USA

Kay-Uwe Wagner

USA

Robert Wagner

USA
Quinten Waisfisz

Netherlands

Stefan Walenta

Germany

Jan Walewski

Poland

Jo Waller

UK

Markus Wallwiener

Germany

Guohui Wan

USA

Bin Wang

USA

Chunmei Wang

USA

Jaw-Yuan Wang

Taiwan

Endi Wang

USA

Fen Wang

USA

Jiucun Wang

China

Jianhua Wang

China

Zhen-Ning Wang

China

Qian Wang

Australia

Li Dong Wang

China

Lu-Hai Wang

Taiwan

Michael Wang

Singapore

Ruoning Wang

USA

Shengzhi Wang

USA

Shouju Wang

China

Kui Wang

China
Tony Wang

USA

Zhen Wang

China

Chen Wang

USA

Anhui Wang

China

Cheng-Ping Wang

Taiwan

Kai Wang

China

De-Shen Wang

China

Ji Ming Wang

USA

Guanghui Wang

China

Jiahong Wang

China

Wen-Lung Wang

Taiwan

Lei Wang

USA

Wei Wang

USA

Xiaoxia Wang

China

Xiao-Si Wang

UK

Zehua Wang

China

Irene Wapnir

USA

Alister Ward

Australia

Doug Ward

UK

John Ward

USA

Fredrik Warnberg Sweden

Sugiko Watanabe Japan 


\begin{tabular}{|c|c|c|}
\hline $\begin{array}{l}\text { Naoki Watanabe } \\
\text { Japan }\end{array}$ & $\begin{array}{l}\text { Hayley Whitaker } \\
\text { UK }\end{array}$ & $\begin{array}{l}\text { Maria Wong } \\
\text { Hong Kong }\end{array}$ \\
\hline $\begin{array}{l}\text { William Watson } \\
\text { Ireland }\end{array}$ & $\begin{array}{l}\text { Emily White } \\
\text { USA }\end{array}$ & $\begin{array}{l}\text { Shun Wong } \\
\text { Canada }\end{array}$ \\
\hline $\begin{array}{l}\text { Elizabeth Wattenberg } \\
\text { USA }\end{array}$ & $\begin{array}{l}\text { Alexandra White } \\
\text { USA }\end{array}$ & $\begin{array}{l}\text { Alex Wong } \\
\text { USA }\end{array}$ \\
\hline $\begin{array}{l}\text { Patrik Weder } \\
\text { Switzerland }\end{array}$ & $\begin{array}{l}\text { David Whiteman } \\
\text { Australia }\end{array}$ & $\begin{array}{l}\text { Wendy Woodward } \\
\text { USA }\end{array}$ \\
\hline $\begin{array}{l}\text { Zheng Wei } \\
\text { China }\end{array}$ & $\begin{array}{l}\text { Emilia Wiechec } \\
\text { Sweden }\end{array}$ & $\begin{array}{l}\text { Chadwick Wright } \\
\text { USA }\end{array}$ \\
\hline $\begin{array}{l}\text { Joanne Weidhaas } \\
\text { USA }\end{array}$ & $\begin{array}{l}\text { Armin Wiegering } \\
\text { Germany }\end{array}$ & $\begin{array}{l}\text { Gerald Wright } \\
\text { USA }\end{array}$ \\
\hline $\begin{array}{l}\text { Kurt Weiss } \\
\text { USA }\end{array}$ & $\begin{array}{l}\text { Charlotte Wilhelm-Benartzi } \\
\text { UK }\end{array}$ & $\begin{array}{l}\text { Qiang Wu } \\
\text { China }\end{array}$ \\
\hline $\begin{array}{l}\text { Aaron Weiss } \\
\text { USA }\end{array}$ & $\begin{array}{l}\text { Stefan Willems } \\
\text { Netherlands }\end{array}$ & $\begin{array}{l}\text { Anhua Wu } \\
\text { China }\end{array}$ \\
\hline $\begin{array}{l}\text { Meng Welliver } \\
\text { USA }\end{array}$ & $\begin{array}{l}\text { Basem William } \\
\text { USA }\end{array}$ & $\begin{array}{l}\text { Cheng-Wen Wu } \\
\text { Taiwan }\end{array}$ \\
\hline $\begin{array}{l}\text { Thilo Welsch } \\
\text { Germany }\end{array}$ & $\begin{array}{l}\text { Anna-Lise Williamson } \\
\text { South Africa }\end{array}$ & $\begin{array}{l}\text { Erxi Wu } \\
\text { USA }\end{array}$ \\
\hline $\begin{array}{l}\text { Shijun Wen } \\
\text { China }\end{array}$ & $\begin{array}{l}\text { Matthew Witek } \\
\text { USA }\end{array}$ & $\begin{array}{l}\text { Jun-Xin Wu } \\
\text { China }\end{array}$ \\
\hline $\begin{array}{l}\text { Thomas G. Wendt } \\
\text { Germany }\end{array}$ & $\begin{array}{l}\text { Sara Wobker } \\
\text { USA }\end{array}$ & $\begin{array}{l}\text { Ning Wu } \\
\text { Canada }\end{array}$ \\
\hline $\begin{array}{l}\text { Rhay-Hung Weng } \\
\text { Taiwan }\end{array}$ & $\begin{array}{l}\text { Thomas Wolf } \\
\text { Germany }\end{array}$ & $\begin{array}{l}\text { Weizhong Wu } \\
\text { China }\end{array}$ \\
\hline $\begin{array}{l}\text { Martin Werner } \\
\text { Germany }\end{array}$ & $\begin{array}{l}\text { Anita Wolfer } \\
\text { Switzerland }\end{array}$ & $\begin{array}{l}\text { Jiong Wu } \\
\text { China }\end{array}$ \\
\hline $\begin{array}{l}\text { Haim Werner } \\
\text { Israel }\end{array}$ & $\begin{array}{l}\text { Hendrik Andreas Wolff } \\
\text { Germany }\end{array}$ & $\begin{array}{l}\text { Junhua Wu } \\
\text { China }\end{array}$ \\
\hline $\begin{array}{l}\text { Kristin Werner } \\
\text { Germany }\end{array}$ & $\begin{array}{l}\text { Aaron H. Wolfson } \\
\text { USA }\end{array}$ & $\begin{array}{l}\text { Minghua Wu } \\
\text { China }\end{array}$ \\
\hline $\begin{array}{l}\text { Jelle Wesseling } \\
\text { Netherlands }\end{array}$ & $\begin{array}{l}\text { Younf-Joo Won } \\
\text { South Korea }\end{array}$ & $\begin{array}{l}\text { Xuping Wu } \\
\text { Sweden }\end{array}$ \\
\hline $\begin{array}{l}\text { Derek West } \\
\text { USA }\end{array}$ & $\begin{array}{l}\text { Young-Woong Won } \\
\text { South Korea }\end{array}$ & $\begin{array}{l}\text { Fen Xia } \\
\text { USA }\end{array}$ \\
\hline $\begin{array}{l}\text { Tamarah Westmoreland } \\
\text { USA }\end{array}$ & $\begin{array}{l}\text { Alice Wong } \\
\text { Hong Kong }\end{array}$ & $\begin{array}{l}\text { Hong-Fei Xia } \\
\text { China }\end{array}$ \\
\hline $\begin{array}{l}\text { Brian Weston } \\
\text { USA }\end{array}$ & $\begin{array}{l}\text { Eric Wong } \\
\text { USA }\end{array}$ & $\begin{array}{l}\text { Jinglin Xia } \\
\text { China }\end{array}$ \\
\hline $\begin{array}{l}\text { Nicholas Wetjen } \\
\text { USA }\end{array}$ & $\begin{array}{l}\text { Kwong-Kwok Wong } \\
\text { USA }\end{array}$ & $\begin{array}{l}\text { Dong Xiao } \\
\text { USA }\end{array}$ \\
\hline $\begin{array}{l}\text { Chery Whipple } \\
\text { USA }\end{array}$ & $\begin{array}{l}\text { Kwan Yeung Wong } \\
\text { Hong Kong }\end{array}$ & $\begin{array}{l}\text { Feifei Xiao } \\
\text { USA }\end{array}$ \\
\hline
\end{tabular}




\begin{tabular}{|c|c|c|}
\hline Guanghua Xiao & Haining Yang & Chueh-Chuan Yen \\
\hline USA & USA & Taiwan \\
\hline Helan Xiao & Jianhua Yang & Chia Jui Yen \\
\hline Canada & USA & Taiwan \\
\hline Jianru Xiao & Jin-Ming Yang & Sriram Yennu \\
\hline China & USA & USA \\
\hline Xiangwei Xiao & Lei Yang & Young Yeom \\
\hline USA & China & South Korea \\
\hline Feng Xiaobin & Lily Yang & Ho-Young Yhim \\
\hline China & USA & South Korea \\
\hline Xu Xinglu & Qiwei Yang & He Yifeng \\
\hline China & USA & China \\
\hline Shunbin Xiong & Ping Yang & James Yiin \\
\hline USA & China & USA \\
\hline Fan Xu & Jinji Yang & John Yim \\
\hline China & China & USA \\
\hline Jia Xu & Ping-Chang Yang & Lu Yin \\
\hline USA & Canada & USA \\
\hline Jiejie Xu & Tsung-Lin Yang & Tinghui Yin \\
\hline China & Taiwan & China \\
\hline Yan Xu & Elizabeth Yanik & Jun Yin \\
\hline USA & USA & China \\
\hline Yh Xu & Qizhi Yao & Michele Yip-Schneider \\
\hline China & USA & USA \\
\hline Yaji Xu & Jian Yao & Takehiko Yokobori \\
\hline USA & Japan & Japan \\
\hline Yasuhide Yamada & Slav Yartsev & Kohei Yokoi \\
\hline Japan & Canada & Japan \\
\hline Toshiyuki Yamaji & Hemad Yasaei & Raymund Yong \\
\hline Japan & UK & USA \\
\hline Yoriko Yamashita & Patsy Yates & Bryan Yoo \\
\hline Japan & Australia & USA \\
\hline Takashi Yamatodani & Ozan Yazici & Kyong-Ah Yoon \\
\hline Japan & Turkey & South Korea \\
\hline Takahiro Yamauchi & Yihong Ye & Go Yoshida \\
\hline Japan & USA & Japan \\
\hline Hideya Yamazaki & Herman Yeger & Junko Yoshida \\
\hline Japan & Canada & Japan \\
\hline Chunhong Yan & Chau-Ting Yeh & Takaki Yoshikawa \\
\hline USA & Taiwan & Japan \\
\hline Dongsheng Yan & Shiou-Hwei Yeh & Tiangeng You \\
\hline China & Taiwan & China \\
\hline Seung Ho Yang & Te-Huei Yeh & Danny Youlden \\
\hline South Korea & Taiwan & Australia \\
\hline
\end{tabular}


Buhyun Youn

South Korea

Jessica Young

USA

Susan Yount

USA

Ramy Youssef

USA

Ramy Youssef Yaacoub

USA

Chao-Lan Yu

USA

Jun Yu Hong

Kong

Guanzhen Yu

China

Helena Yu

USA

Huiqing Yuan

China

Guangwen Yuan

China

Zheng Yuan

China

Yuan Yuan

China

Yuan Yuan

USA

Wei Yue

USA

Xiaoqiang Yue

China

Jong Won Yun

South Korea

Jason Yustein

USA

Aziz Zaanan

France

Iris Zachary

USA

Fabrizio Zanconati

Italy

Roberto Zanetti

Italy
Uwe Zangemeister-Wittke

Switzerland

Marjorie Zauderer

USA

Robert Zeillinger

Austria

Alain Zeimet

Austria

Zihua Zeng

USA

Jiping Zeng

China

Zhi Zeng

China

Zhirong Zeng

China

Pio Zeppa

Italy

Alessandro Zerbi

Italy

Baolin Zhang

USA

Faming Zhang

China

Hong (Amy) Zhang

USA

Jinsheng Zhang

China

Jun Zhang

China

Junran Zhang

USA

Ming Zhang

USA

Siyuan Zhang

USA

Weiguo Zhang

USA

Xiaoyu Zhang

USA

Kunhe Zhang

China

Yi Zhang

USA
Mei Zhang

USA

Guangjun Zhang

China

Guo Zhang

China

Lining Zhang

China

Lin Zhang

USA

Meixia Zhang

China

Mei-Yun Zhang

Hong Kong

Xuegong Zhang

China

Zhen Zhang

USA

Zhongzu Zhang

China

Zhi-Gang Zhang

China

Chen Zhao

USA

Qichen Zhao

China

Weili Zhao

China

Guoqiang Zhao

China

Hongyun Zhao

China

Minhua Zheng

China

Yongqiu Zheng

USA

Yuanyi Zheng

China

Qiaoming Zhi

China

Yuan Zhiyong

China

Weide Zhong

China 
Bo Zhou

China

Qingyu Zhou

USA

Shu-Feng Zhou

USA

Tong Zhou

USA

Yu-Hao Zhou

China

Jingjiao Zhou

USA

Jinfeng Zhou

China

Jue-Yu Zhou

China

Yifeng Zhou

China

Jingde Zhu

China

Ji Zhu

China
Yueming Zhu

USA

Zhitu Zhu

China

Zhengping Zhuang

USA

Suzanna Zick

USA

Andries Zijlstra

USA

Volker Ziller

Germany

Michael Zilliox

USA

Yitzhak Zimmer

Switzerland

Daniel Zips

Germany

Mateo Ziu

USA

Oliver Zivanovic

USA
Inti Zlobec

Switzerland

Anna Zolkiewska

USA

Gabriele Zoppoli

Italy

Xiaoping Zou

China

Chiara Zuiani

Italy

Mingxin Zuo

USA

Wilbert Zwart

Netherlands

Ellen Zwarthoff

Netherlands

Krzysztof Zwierz

Poland

Norberto Walter Zwirner

Argentina 